\title{
Reynald Rivard, prêtre psychologue trifluvien, l'essor de l'éducation spécialisée au Québec et la fin des orphelinats ordinaires (1947-1968)
}

\section{Lucia Ferretti}

Volume 62, numéro 3-4, hiver-printemps 2009

La culture catholique

URI : https://id.erudit.org/iderudit/038524ar

DOI : https://doi.org/10.7202/038524ar

Aller au sommaire du numéro

\section{Éditeur(s)}

Institut d'histoire de l'Amérique française

ISSN

0035-2357 (imprimé)

1492-1383 (numérique)

Découvrir la revue

Citer cet article

Ferretti, L. (2009). Reynald Rivard, prêtre psychologue trifluvien, l'essor de l'éducation spécialisée au Québec et la fin des orphelinats ordinaires (1947-1968). Revue d'histoire de l'Amérique française, 62(3-4), 533-564. https://doi.org/10.7202/038524ar

\section{Résumé de l'article}

Reynald Rivard est un pionnier de l'éducation spécialisée au Québec. Trois constats peuvent être tirés de sa biographie professionnelle. Sans remettre en question l'apport du réseau constitué autour de Boscoville à l'émergence de l'éducation spécialisée, on s'aperçoit qu'au milieu du $\mathrm{xx}^{\mathrm{e}}$ siècle la réflexion sur ce champ d'intervention et de formation a été plus générale et qu'elle a puisé à des courants théoriques et pratiques contrastés, voire opposés. Par ailleurs, l'article documente des aspects essentiels de la déqualification subie par les religieuses après 1950 : l'impossibilité de faire valider leur formation professionnelle par un diplôme reconnu, ainsi que le refus des autorités cléricales et étatiques de leur faciliter l'accès aux formations habilitant aux titres professionnels. Enfin, au-delà des relations entre l'Église et l'État, la mise en évidence des rapports de forces présents au sein même de chacune de ces institutions contribue à rendre toute sa complexité au processus ayant abouti à la disparition des orphelinats ordinaires.
Tous droits réservés @ Institut d'histoire de l'Amérique française, 2009
Ce document est protégé par la loi sur le droit d'auteur. L'utilisation des services d'Érudit (y compris la reproduction) est assujettie à sa politique d'utilisation que vous pouvez consulter en ligne. 


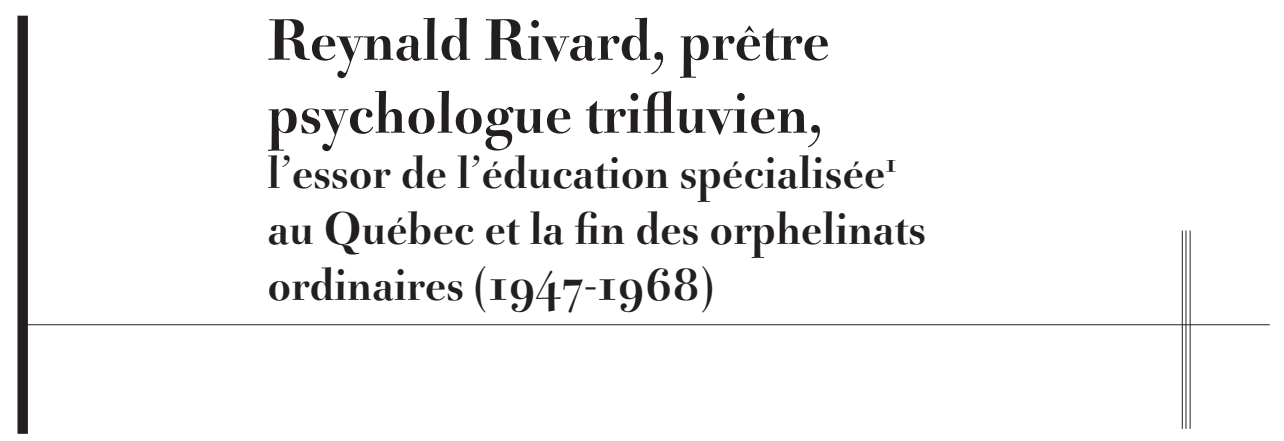

LUCIA FERRETTI

Département des sciences humaines/CIEQ

Université du Québec à Trois-Rivières ${ }^{2}$

RÉSUMÉ - Reynald Rivard est un pionnier de l'éducation spécialisée au Québec. Trois constats peuvent être tirés de sa biographie professionnelle. Sans remettre en question l'apport du réseau constitué autour de Boscoville à l'émergence de l'éducation spécialisée, on s'aperçoit qu'au milieu du $x x^{e}$ siècle la réflexion sur ce champ d'intervention et de formation a été plus générale et qu'elle a puisé à des courants théoriques et pratiques contrastés, voire opposés. Par ailleurs, l'article documente des aspects essentiels de la déqualification subie par les religieuses après 1950: l'impossibilité de faire valider leur formation professionnelle par un diplôme reconnu, ainsi que le refus des autorités cléricales et étatiques de leur faciliter l'accès aux formations habilitant aux titres professionnels. Enfin, au-delà des relations entre l'Église et l'État, la mise en évidence des rapports de forces présents au sein même de chacune de ces institutions contribue à rendre toute sa complexité au processus ayant abouti à la disparition des orphelinats ordinaires.

ABSTRACT - Reynald Rivard was a pioneer in special education in Quebec. Three conclusions can be drawn from his professional biography. Without questioning the contribution

1. Dans les années 1940 à 1970, la terminologie n’est pas définitive. Éducation spécialisée, socio-pédagogie, psychopédagogie et, plus tard, psychoéducation: autant d'expressions utilisées alors par ceux qui sont en train d'élaborer un champ d'interventions auprès des jeunes en difficulté conjuguant les objectifs de l'éducation proprement dite et ceux de la psychologie.

2. J'exprime ma chaleureuse gratitude à madame Anita Cadotte, qui a constitué le fonds ReynaldRivard pendant une cinquantaine d'années et l'a déposé au Service des archives du Séminaire SaintJoseph (cote FN 0733, non indexé). Merci aussi à mes collègues René Hardy, Jean Roy et Jocelyne Moreau ainsi qu'aux évaluateurs anonymes pour leurs commentaires si pertinents. 
made by the network developed around Boscoville to the emergence of special education, there was a broader reflection on the field and the training of professionals in the mid- $20^{\text {th }}$ century, one which drew on diverse, and sometimes conflicting, theoretical and practical currents. Furthermore, the article describes the essential elements of the marginalization of religious orders after 1950: their inability to support their professional training with recognized diplomas, as well as the refusal of church authorities and government to help them access the training necessary to gain professional accreditation. Finally, looking beyond the relationship between Church and State, an examination of the power relationships within each of these institutions helps to reveal the fundamental complexity of the process which led to the disappearance of orphanages.

D ans un mouvement qui, un peu après ce qu'on observe en milieu protestant, touche à leur tour les pays et sociétés catholiques en Occident pendant ou après la Seconde Guerre mondiale, plusieurs professions liées au grand secteur de la protection de la jeunesse apparaissent ou prennent alors leur essor au Québec. On pense à celles que pratiquent les travailleurs sociaux, les psychologues ou les criminologues par exemple ${ }^{3}$. Issues du terreau de la doctrine sociale de l'Église, ces professions se fondent beaucoup sur la psychologie, qui s'impose de plus en plus à cette époque comme méthode de compréhension des problèmes de l'enfance et de leur traitement ainsi que méthode de normalisation des comportements.

L’éducation spécialisée est un autre de ces champs apparentés d’intervention et de formation qui se constitue dans les années 1940 à 1970. Jusqu’à récemment, nous ne pouvions compter que sur les pionniers de

3. Depuis l'ouvrage de Lionel-H Groulx, Le travail social. Analyse et évolution, débats et enjeux (Laval, Éditions Agence d'Arc, 1993), 297 p., les études se sont multipliées sur l'histoire du travail social au Québec. Parmi les plus récentes, Denyse Baillargeon, Amélie Bourbeau et Gilles Rondeau, "Motivation et formation des travailleurs sociaux francophones: quelques parcours pionniers (1942-1961)", Intervention, 125 (2006): 169-179; et Julien Prud'homme, "L'expertise professionnelle et l'État-providence. Les travailleurs sociaux québécois et la "technocratisation" du service social, 1970-2000", Revue d'histoire de l'Amérique française, 62,1 (hiver 2009): 95-109. Ces articles mentionnent plusieurs références québécoises et internationales que le manque de place m’empêche de citer à mon tour. C'est le cas aussi pour les titres suivants. Roger Tessier, "L'intervention psychosociologique de 1940 à 1990: historique et essai de clarification conceptuelle», dans R. Tessier et Y. Tellier, dir., Changement planifié et développement des organisations. Historique et prospective de changement planifié (Québec, Presses de l’Université du Québef, 1990), 1: 89-113; Julien Prud'homme, «Professionnelles des soins et marchés de la santé. Les trajectoires analogues des physiothérapeutes et des psychologues québécoises, 1950-2010", RHAF, 62,2 (automne 2008): 253-287; Marcel Fournier, Entretiens avec Denis Zsabo. Fondation et fondements de la criminologie (Montréal, Liber, 1998), 227 p.; Simonne Landry, "Psychosociologie et relations humaines à l'Université de Montréal et à l’Université du Québec à Montréal à vol d'oiseau ", Interactions, 7,1 (printemps 2003): 14-16. 
Boscoville pour nous rappeler les débuts de cette profession au Québec, avec les biais inévitables qu'une telle posture induit ${ }^{4}$. On pense aux travaux de Gilles Gendreau par exemple, et à ceux qu'il a suscités ${ }^{5}$. La naissance de l'éducation spécialisée y apparaît liée à un problème spécifique, la délinquance juvénile; à un type d'établissements: Boscoville lui-même, grand complexe pavillonnaire sans clôture construit au tournant des années 1950 au bord de la rivière des Prairies, à Montréal, en réaction contre les internats décrits comme tristes, désuets et désargentés où on continue d'ailleurs à enfermer les jeunes; à une conception de la délinquance non plus comme perversion morale à combattre mais comme blessure psychique à guérir, et en conséquence à des objectifs de traitement qui ne sont plus la réadaptation à la société mais la rééducation de la personnalité; à une thérapie de type analytique conçue à l'Institut de psychologie de Montréal et au Centre d'orientation lié à Boscoville, et qui est enseignée à l'Université de Montréal à partir de 1954; et, enfin, à un nouveau genre de praticiens: non plus des religieux, mais des jeunes hommes laïques qui font de leur propre personnalité équilibrée leur principal outil professionnel.

Cet article s'inscrit dans la foulée des travaux qui, sans minimiser le rôle de ces acteurs ni de ces établissements, ont commencé depuis quelques années à problématiser Boscoville autrement ainsi qu’à démontrer que l'éducation spécialisée pousse des racines plus anciennes et plus étendues qu'on ne l'a reconnu jusqu'ici. Louise Bienvenue, par exemple, inscrit l'action psychoéducative conduite à Boscoville dans l'histoire des régulations sociales et se demande notamment si la thérapeutique invasive qui y était pratiquée n'était pas tout compte fait au moins aussi contrôlante que quelques-unes des méthodes employées ailleurs par les frères ${ }^{6}$. Par

4. En France aussi, des praticiens-chercheurs ont eu à cœur de préserver la mémoire des débuts de l'éducation spécialisée, qu'ils font remonter au moment où eux-mêmes sont entrés dans la carrière. Conservatoire national des archives et de l'histoire de l'éducation spécialisée, Elles ont épousé l'éducation spécialisée. Éducatrices et femmes d'éducateurs il y a cinquante ans (Paris, L’Harmattan, 1999), 224 p., textes choisis et présentés par Mathias Gardet, Vincent Peyre et Françoise Tétard.

5. Gilles Gendreau, avec la collaboration de Diane Métayer et André Lebon, L'action éducative. Pour qui? Pour quoi? (Paris, éditions Fleurus, 1990), 36-52; Jean Ducharme, Saute d'abord! Un parcours de trente-cinq ans en psychoéducation (Montréal, Éditions Sciences et culture, 1999), 217 p.; Marie-Paule Leduc, Ces enfants qui m'ont tant appris. Expérience d'hier... source d'inspiration pour aujourd'hui (Montréal, Éditions Sciences et culture, 2003), 171 p. (notamment l'avant-propos de Francine Béchard). Voir aussi Marcel Renou, «La psychoéducation: une perspective historique», Revue canadienne de psycho-éducation, 18,2 (1989): 63-88.

6. Louise Bienvenue, «Je t’observe, mais je m'observe aussi. Nature du lien psychoéducatif à Boscoville, 1950-1970", communication au séminaire Jeunesse et régulations sociales. Regards comparés Canada-France, $19^{\mathrm{e}}-20^{\mathrm{e}}$ siècles, Orford, juin 2009. À paraître sous le titre «Un tournant dans l'histoire 
ailleurs, dans l'important ouvrage dirigé par Marie-Paule Malouin, on se montre très attentif à sortir de l'ombre les tentatives de certaines congrégations religieuses, notamment féminines, pour améliorer leur intervention auprès des enfants qu'elles hébergeaient dans leurs grandes maisons ${ }^{7}$. Pour notre part, nous étudions ici le parcours de l'abbé Reynald Rivard. Par la mise au jour de certains éléments de sa biographie professionnelle ${ }^{8}$, nous visons deux objectifs: faire connaître la contribution encore ignorée de celui-ci à l'essor de l'éducation spécialisée au Québec, mais surtout resituer les origines de ce champ d'intervention et de formation dans toute la complexité du contexte de transition qui marque alors la réflexion et les pratiques entourant la prise en charge des enfants dits désavantagés, exceptionnels ou inadaptés.

Né en 1919 à Trois-Rivières, Reynald Rivard commence dès son ordination à la prêtrise en 1944 à travailler étroitement avec l'abbé CharlesÉdouard Bourgeois, le grand organisateur des services à l'enfance sans soutien dans le diocèse de Trois-Rivières ${ }^{9}$. De 1947 à 1968, il est à la fois assistant aumônier à l'orphelinat pour les garçons de 6 à 12 ans, directeur de la clinique d'hygiène mentale, et directeur adjoint de l'agence diocésaine de service social. Comme spécialiste de la psychologie de l'enfance appliquée à l'éducation, il a défendu une approche de l'éducation spécialisée radicalement différente de celle sur laquelle on misait à Boscoville, au Centre d'orientation et à l'Université de Montréal. Sans doute, le milieu était-il tout autre: le prêtre psychologue avait à se préoccuper principalement de garçons de moins de 12 ans, placés dans ce qu'on appelait alors des orphelinats ordinaires tenus par des religieuses. Adepte de la psycho-

des régulations sociales. La "rééducation totale" des délinquants à Boscoville (1942-1970)", Recherches sociographiques. On y trouve plusieurs références québécoises et internationales à l'histoire de la psychologie au milieu du $\mathrm{xx}^{\mathrm{e}}$ siècle que je ne reprendrai pas ici.

7. Marie-Paule Malouin, dir., L'univers des enfants en difficulté au Québec entre 1940 et 1960 (Montréal, Bellarmin, 1996), 458 p. Un mouvement semblable s'est produit dans l'historiographie européenne. Monique Vial, Les enfants anormaux à l'école. Aux origines de l'éducation spécialisée, 1882-1909 (Paris, Armand Colin, 1990), 231 p.; Claude Wacjman, L'enfance inadaptée. Anthologie de textes fondamentaux (Toulouse, Privat, 1993), 214 p. ; Marcel Jaeger, Aux sources de l'éducation spécialisée, 1878-1910: la formation des premières infirmières laïques (Paris, Éditions du CTNERHI, 1998), 147 p. ; Jean-Louis Korpes, Handicap mental. Notes d'histoire (Lausanne, Éditions de l'EESP, s.d. (après 1987)), $67 \mathrm{p}$.

8. D’autres aspects de sa carrière dans Lucia Ferretti et Chantal Bourassa, «Reynald Rivard: un psychologue trifluvien au service de l'enfance exceptionnelle (1947-1968)", dans Jean Roy et Lucia Ferretti, dir., Nouvelles pages trifluviennes (Sillery, Septentrion, 2009), 121-147.

9. L. Ferretti, «Charles-Édouard Bourgeois, prêtre trifluvien, et les origines diocésaines de l'État-providence au Québec (1930-1960)", Nouvelles pratiques sociales, 14,1 (juin 2001): 169-182. 
logie sociale expérimentale, il se méfiait des thérapies de type analytique. Il voulait moins réformer la personnalité des jeunes que leur permettre de l'exprimer. Il voulait aussi les aider à se façonner des comportements acceptables par la société. À cette fin, il a développé un type d'intervention fondé sur les loisirs et sur le rôle du groupe dans l'adaptation de chacun à la vie sociale. Aux religieuses, il a été soucieux de fournir une première formation professionnelle, qu'il a développée avec l'Université d'Ottawa. Puis, à l'intention surtout des hommes laïques, il a participé à l'élaboration d'une formation d'éducateur spécialisé fondée sur l'enseignement de la dynamique de groupe et qui a été donnée entre autres au Centre d'études universitaires de Trois-Rivières.

Dans les années 1940 à 1970, l'éducation spécialisée se constitue au confluent de multiples enjeux. Marie-Paule Malouin a bien mis en valeur que c'est non seulement comme religieuses mais comme femmes que les sœurs sont marginalisées dans le mouvement d'étatisation, de sécularisation et de masculinisation de l'éducation et du bien-être social caractéristique de ces années. L'éducation spécialisée se taille alors un nouveau chemin à travers les rapports de force entre Église et État, mais peut-être davantage encore, comme nous le verrons, entre centres de services sociaux et institutions au sein de l'Église, et entre le secteur de l'éducation et celui du bien-être social au sein de l'État. Une partie du destin des orphelinats ordinaires et des religieuses éducatrices se joue là : dans les rivalités interprofessionnelles entre travailleurs sociaux et éducateurs spécialisés ainsi que dans le contraste entre le dynamisme du département de l'Instruction publique puis du ministère de l'Éducation d'une part, et, d'autre part, le traditionalisme du ministère de la Famille et du Bien-être social. L'éducation spécialisée évolue aussi sous l'effet de pressions internes: oppositions entre les théories psychologiques et les conceptions de l'intervention auprès des jeunes, disproportion entre les établissements universitaires qui proposent les formations. C'est l'ensemble de ces facteurs qui expliquent qu'à terme le travail de Reynald Rivard soit resté presque sans suite.

Les archives personnelles du prêtre psychologue composent un fonds de 38 boîtes, dont presque la moitié touchent de près ou de loin l'éducation des enfants en difficulté. On y trouve essentiellement des conférences, des procès-verbaux, de la correspondance, des brochures, des rapports divers, des photographies, des notes personnelles. En fait, tout semble avoir été conservé, dans l'ordre et selon les critères établis au moment de la constitution du fonds. Ces qualités exceptionnelles rendent possible de 
reconstituer la nature et le sens du projet éducatif de l'abbé Rivard et de situer sa réflexion et son action au confluent des nombreuses influences qui les conditionnent.

Cet article est donc une contribution à l'histoire des origines de l'éducation spécialisée au Québec. Il comprend quatre parties. Nous présentons d'abord le milieu dans lequel exerce le jeune prêtre, les influences qui le modèlent et ses conceptions de l'enfant et de l'éducation. Or, son action s'inscrit dans le contexte d'après-guerre, au cours duquel, de nouveaux paramètres guidant la réflexion collective sur les milieux les plus favorables au développement des enfants, l'abbé Rivard entreprend de réformer en profondeur les orphelinats ordinaires de Trois-Rivières. Un aspect particulièrement important de cette réforme concerne la préparation professionnelle des éducatrices puis des éducateurs spécialisés. Mais malgré de réels succès, reconnus à l'échelle du Québec et au-delà, l'effort de Rivard s'inscrit à contre-courant des tendances de fond des années 1950 et surtout 1960, qui mèneront à la fin des orphelinats ordinaires.

\section{I - L'ENFANT EST UNE PERSONNE, ET L'ÉDUCATION DOIT ÊTRE ORGANISÉE EN FONCTION DE SES BESOINS}

En 1947, quand Reynald Rivard devient l'adjoint de l'abbé CharlesÉdouard Bourgeois à l'Assistance à l'enfant sans soutien, cette agence sociale est en train de se doter d'un nouveau service: une clinique de psychologie et d'orientation, qui en sera détachée l'année suivante et prendra le nom d'Institut psycho-social (IPS) ${ }^{10}$. À ses débuts, l'IPS se donne un mandat de dépistage et de traitement des troubles mentaux des enfants "mésadaptés, difficiles et anormaux » qui vivent dans leur propre famille ou qui, pour ces raisons ou d'autres, ont été placés dans les établissements d'assistance publique de Trois-Rivières ${ }^{11}$. C'est qu'on s'aperçoit bien, et tout particulièrement Rivard en tant qu'assistant aumônier à l'Orphelinat Saint-Dominique, que les jeunes qui arrivent en institution et y grandissent sont carencés, en particulier sous le rapport du développement de leur personnalité et de leur adaptation à la vie en société.

À l'IPS, toute l'approche est fondée sur la psychologie expérimentale, sur ses méthodes et sur ses objectifs. Pour bien comprendre un enfant et intervenir de manière pertinente auprès de lui, il faut le connaître, d'où

10. «Fondation de l'Institut psycho-social des Trois-Rivières, une œuvre pour enfants infirmes ou déficients ", Le Nouvelliste, 18 novembre 1948, 3.

11. Rapport annuel 1949, Trois-Rivières, Institut psycho-social, 1949, 16 p. Ce rapport et presque tous les suivants sont conservés à la bibliothèque de l’UQTR. 
les tests qu'on lui fait passer en vue de le classer soit parmi les enfants normaux, soit dans l'une ou plusieurs des multiples catégories de l'enfance exceptionnelle: sous-doués, surdoués, handicapés physiques, sociaux ou affectifs, arriérés pédagogiques, mésadaptés, etc. ${ }^{12}$. Quant à la thérapeutique, on entre alors, en Europe comme en Amérique du Nord, dans une époque qui va durer une bonne trentaine d'années, tout au long de laquelle celle-ci se fonde essentiellement sur des soins spécialisés dispensés à l'écart des enfants ordinaires ${ }^{13}$. À l'IPS, le traitement consiste habituellement en séances de psychothérapie par le groupe, et plus rarement par voie directe (individuelle). On y oriente aussi les arriérés pédagogiques vers des classes spéciales lorsqu'elles existent, et on transfère les déficients mentaux vers des instituts spécialisés, tous situés en dehors de la région. Ce faisant, on veut aider chaque jeune à développer son plein potentiel, alléger la tâche des familles dans l'espoir qu'elles seront moins tentées de placer leurs enfants handicapés ou difficiles, sortir des classes régulières les élèves qui freinent le groupe et désengorger les orphelinats ordinaires des cas les plus lourds ${ }^{14}$.

À peine embauché, l'abbé Rivard entreprend des études à l'Université d'Ottawa ${ }^{15}$, où $1^{\prime}$ Institut de psychologie dirigé par le père Raymond

12. Pour d'autres exemples de classification, L'enfance exceptionnelle. Mémoire présenté à la Commission royale d'enquête sur l'éducation par le Conseil des ouvres de Montréal, avril 1962, 18-19, et Rapport de la Commission royale d'enquête sur l'enseignement dans la Province de Québec (Rapport Parent), 2: 481-525. Sur l'emploi des tests auprès des enfants au Québec, Martin Pâquet et Jérôme Boivin, «La mesure fait loi. La doctrine de l'hygiène mentale et les tests psychométriques au Québec pendant l'entre-deux-guerres", Canadian Historical Review, 88,1 (2007): 149-178; y sont notamment citées plusieurs études concernant le monde anglo-saxon.

13. Nathalie Bélanger, De la psychologie scolaire à la politique de l'enfance inadaptée (Paris, Éditions du CTNERHI, 2002), 148 p. (notamment le chapitre 1).

14. Dès 1944, dans son mémoire devant la Commission d'assurance-maladie, l'abbé Bourgeois avait plaidé en ce sens. "Centrale provinciale de nos œuvres d'assistance publique», Le Nouvelliste, 9 mars 1944, 3, et «Rapport annuel de l'Assistance à l'enfant sans soutien, 1944», Le Nouvelliste, 27 février 1945, 3. À la fin des années 1940, le système québécois de prise en charge des enfants exceptionnels, du côté catholique, comprend un secteur peu développé de placement familial, mais en revanche plusieurs établissements. D'une part les orphelinats ordinaires, les écoles d'industrie et les écoles de réforme (celles-ci seront regroupées en 1950 pour devenir des écoles de protection de la jeunesse). S'y ajoutent, d'autre part, des institutions plus spécialisées. Pour les déficients mentaux, plusieurs internats, un externat, l'école Victor-Doré de la Commission des écoles catholiques de Montréal, et les hôpitaux psychiatriques; d'autres institutions pour aveugles et pour sourds-muets. Outre l'ouvrage de Marie-Paule Malouin déjà cité, voir Lettre de Louis-Philippe Audet, directeur des études au ministère du Bien-être social, aux membres du Comité d'études sur l'enfance exceptionnelle, 14 mars 1960 (ASTR, FN 0733, Boîte 8, chemise «Éducation de l'enfance exceptionnelle. Formation des éducateurs». Désormais les références seront notées sous la forme B. ${ }^{\circ}$, ch. «titre».)

15. Lettre de l'abbé Charles-Édouard Bourgeois à $\mathrm{M}^{\mathrm{gr}}$ Georges-Léon Pelletier, évêque de Trois-Rivières, 20 septembre 1948 (B. $25,1^{\text {ère }}$ ch. sans titre). 
Shevenell, o.m.i., est reconnu pour son approche psychométrique, adoptée à l'IPS ${ }^{16}$. En 1946, Fred Brown y a mis au point le test Brown-Ottawa sur la personnalité. Ce test, adapté aux enfants de 9 à 14 ans, repose sur une théorie de la névrose comme ayant une origine psychologique et sociale ne devant rien aux conceptions freudiennes de la sexualité infantile ni à la psychanalyse en général ${ }^{17}$. Cela convient au jeune prêtre, réticent devant l'approche clinique-psychanalytique que le dominicain Noël Mailloux est en train de développer à l'Institut de psychologie de l'Université de Montréal $^{18}$. Rivard obtient une licence en 1949, une maîtrise en 1951, et un doctorat en 1955. Son mémoire puis sa thèse sont consacrés à ce qui lui apparaît d'emblée comme la source fondamentale des problèmes des enfants placés en institution, à savoir leur grande instabilité émotive, et aux moyens d'au moins atténuer celle-ci ${ }^{19}$.

C'est donc d'abord son expérience quotidienne auprès des enfants en difficulté, à l'orphelinat et à la clinique, qui nourrit les conceptions de l'enfance, de l'éducation et de l'éducation spécialisée que développe progressivement le prêtre psychologue. Mais celles-ci puisent aussi à une autre source importante. Par $\mathrm{M}^{\mathrm{gr}}$ Bourgeois, qui en devient le vice-président en 1952, Rivard est en effet coopté d'emblée dans le réseau de réflexion et d'échanges qu'est le Bureau international catholique de l'enfance (BICE) $)^{20}$. Pendant plusieurs années, il entretiendra un contact constant et fructueux avec les spécialistes associés à cet organisme, que ce soit par correspondance, collaboration scientifique, visites d'établissements spécialisés en

16. Université d'Ottawa, Faculté des Arts, Annuaire 1950-1951; Université d'Ottawa, École des gradués, Programme des études, 1950-1951. Ces deux documents sont conservés dans le fonds 77, boîte 10-654.

17. Fred Brown, Personnality Inventory for Children (Ottawa, Les éditions de l'Université d'Ottawa, 1946), traduit et adapté sous la direction de R.-H. Shevenell, o.m.i., (B. 11, ch. «Tests et techniques. L'inventaire de la personnalité Brown-Ottawa»).

18. Université de Montréal, Fonds du Département de psychologie (E0023) GE0023010001. Annuaire 1950-1951, 1, 34 p. L'abbé Rivard a conservé des documents mettant en garde contre l'approche psychanalytique: «Le problème moral et la psychanalyse», dans Revue dominicaine, décembre 1947 (B. 11, ch. "Psychologie et morale»); Avertissement du Saint-Office, 19 juillet 1961 (B. 23, ch. «Divers»).

19. R. Rivard, L'instabilité émotionnelle chez les protégés des orphelinats ordinaires de la province de Québec, thèse présentée en vue de la maîtrise ès arts, Université d'Ottawa, 1951, 69 p. ; R. Rivard, L'instabilité émotive dans les grands orphelinats de la province de Québec, thèse de doctorat, Université d'Ottawa, 1955, $132 \mathrm{p}$.

20. Fondé en France en 1947, le BICE a été reconnu en 1948 par le Saint-Siège comme organisation catholique internationale vouée à l'étude de l'enfance sous tous ses aspects, sauf celui strictement scolaire, et s'est vu offrir un statut consultatif auprès du Conseil économique et social des Nations Unies et auprès de l'UNESCO. Voir $3^{e}$ de couverture de son bulletin L'enfance dans le monde; quelques exemplaires en sont conservés dans la b. 8. 
Europe, échange de documentation, ou participation à divers colloques et congrès.

Irriguée par la doctrine sociale de l’Église en redéfinition à cette époque sous l'influence entre autres du personnalisme, par les disciplines montantes que sont la psychologie et la psychopédagogie ainsi que par les principes en voie d'être codifiés dans la Déclaration des droits de l'enfant qu'adoptera l'ONU en 1959 et à la préparation de laquelle le BICE est en train de collaborer, la réflexion de Reynald Rivard s'inscrit dans cette mouvance internationale de valorisation de l'enfant comme personne. «L'enfant est une personne, avec des droits, dont celui que les décisions soient prises en fonction de lui»; «L'enfant est une fin, et ne peut être traité en moyen : la famille, l'Église, l'État doivent viser directement à son bien et non se servir de lui»; éduquer un enfant, c'est le préparer à la «liberté» et viser à le rendre «réellement heureux plus tard»: de telles assertions reviennent chez lui comme un leitmotiv ${ }^{21}$. Être faible et dépendant au départ, l'enfant est néanmoins poussé par sa nature même à s'élancer dans la vie à la poursuite d'un bien, d'une ambition, d'une joie qui lui sont propres. Sa famille et la société doivent lui procurer, par une "éducation intégrale», ce qui lui est nécessaire pour atteindre ses propres fins naturelles et surnaturelles et répondre ainsi au plan personnel que Dieu a tracé pour lui.

Ainsi, là où un $\mathrm{M}^{\mathrm{gr}}$ Bourgeois, par exemple, continue de justifier les ressources publiques qu'il réclame pour l'éducation et la rééducation des jeunes en difficulté surtout dans la perspective de faire d'eux des citoyens utiles et respectueux de l'ordre social ${ }^{22}$, Rivard déplace l'objectif, et c'est plutôt au nom du devoir des parents et de leurs auxiliaires, l'Église et l'État, d'aider chaque enfant à se réaliser qu'il promeut une organisation de l'éducation en fonction des besoins de chacun ${ }^{23}$.

21. Plusieurs exemples dans B. 16, ch. «Cours de perfectionnement aux religieuses de Ville-Joie Saint-Dominique, 11-15 août 1952»; Journée d'étude pour les prêtres des séminaires du diocèse de TroisRivières, du 28 juin au 3 juillet 1954 au Séminaire Saint-Joseph (B. 11, ch. «Psycho-pédagogie Cours aux prêtres éducateurs"); et R. Rivard et Gilles Lacroix, Essai de normes professionnelles des foyers nourriciers (Montréal, Caritas-Canada, 1958), 64 p. (B. 1, ch. «Foyers nourriciers - Standards professionnels").

22. Charles-Édouard Bourgeois, Une richesse à sauver: l'enfant sans soutien (Trois-Rivières, Éditions du Bien public, 1947), 256 p.

23. Rivard expose sa conception de l'enfance à de multiples occasions. Voir entre autres: Vers une éducation intégrale, un film de la Ciné-photographie, direction technique : Reynald Rivard, ptre, de l'Institut psycho-social; réalisation et textes: Michel Vergnes; prises de vue: Fernand Rivard [tout début des années 1950]; «Exposé général des besoins des enfants désavantagés », CaritasCanada, Deuxième congrès - 1954 (Montréal, Caritas-Canada, 1954), 79-85; "Classification des 
Soutenir la famille naturelle dans sa tâche d'élever ses enfants normaux et spéciaux, et, en cas d'incapacité de celle-ci, procurer aux jeunes un milieu de vie substitut approprié et qualifié, c'est ce à quoi s'emploie le prêtre psychologue.

\section{2 - DES ORPHELINATS POUR LES ENFANTS}

\section{I Famille naturelle, foyers nourriciers, institutions : les nouveaux paramètres de la réflexion}

Après la guerre, avec l'émergence de professionnels tels que les psychologues et les travailleurs sociaux, la famille naturelle commence à être l'objet d'une plus grande sollicitude. Elle apparaît de plus en plus comme le milieu le plus propice à l'éducation des enfants. Les intervenants catholiques sur le terrain admettent désormais l'importance de toutes les mesures publiques et privées susceptibles de contrer la maladie et la pauvreté des parents, facteurs reconnus de dislocation des familles; et de toutes les autres qui outillent celles-ci à mieux prendre soin de leurs enfants normaux et exceptionnels ${ }^{24}$. De même, tous, en principe, favorisent l'adoption précoce des enfants abandonnés, une manière de leur fournir pour ainsi dire une nouvelle famille naturelle ${ }^{25}$. Alors que le placement peut être désiré par les parents soit en raison de l'enfant lui-même, soit à cause de leur propre situation et qu'il est une d'habitude bien ancrée dans la population, on cherche donc désormais à contrer cette culture ${ }^{26}$.

déficients", Caritas-Canada, "Caritas Christi urget nos", Troisième congrès - 1955 (Montréal, Université de Montréal, 1955), 135-142. Il termine souvent ses conférences par cette pensée d'un auteur non identifié : «Vous pouvez leur donner votre amour, mais non pas vos pensées, parce qu’ils ont leurs pensées propres. Vous pouvez loger leurs corps mais non pas leurs âmes parce que leurs âmes habitent dans la maison de demain que vous ne pouvez visiter, pas même en rêve. Vous pouvez vous efforcer d'être semblables à eux mais n'essayez pas de les rendre tel que vous êtes. En effet, la vie ne va pas à reculons ni ne s'accroche au passé. Vous êtes comme des arcs par lesquels vos enfants comme des flèches vivantes seront lancés dans l'avenir.» (B. 16, ch. "Cours de psychopédagogie aux Sœurs St-Joseph de St-Vallier - 1952-1958»).

24. Pour le BICE: B. 9, ch. " $2^{\text {ème }}$ congrès 1964, Conseil du Québec de l'enfance exceptionnelle». Les thèmes retenus aux congrès annuels de Caritas-Canada par sa commission «Famille» et sa commission «Enfance» sont aussi très explicites. Pour le Conseil canadien du bien-être social: Rapport de la Commission II..., op. cit.

25. Caritas-Canada, commission "Enfance», Adoption, normes professionnelles (Montréal, Secrétariat national, 1963), 102 p. Avant publication, Rivard a évalué la version finale de ce document (B. 3, ch. "Adoption»).

26. On trouve plusieurs traces de cette culture du placement dans le fonds Rivard. Par exemple, dans le Rapport de la Commission II de la Commission française du Conseil canadien du Bien-être social en novembre 1953 (B. 1, ch. «Foyers nourriciers. Standards professionnels»). Aussi, Romuald Voyer, t.s., "Les réalisations actuelles dans le domaine de la prévention en faveur de l'enfance et de la jeunesse », Caritas-Canada, "Ut sint unum», Quatrième congrès - 1956, Compte rendu des conférences (Montréal, Secrétariat national, 1956), 103-112; Caritas-Canada, "La charité dans la charité», 
On s'entend aussi pour soutenir que lorsque des enfants «normaux» doivent quitter leur foyer ou n'en ont pas, ils devraient être placés dans un milieu qui ressemble le plus possible à une famille. Quel est ce milieu? Là commencent les discussions.

Le prêtre psychologue poursuit sur cette question une réflexion qui dure plus de 20 ans. Il convient sans réserve que dans certaines situations et pour certains enfants, les plus jeunes notamment, le placement familial s'impose. Globalement, toutefois, il ne se départira jamais de sa méfiance à l'égard de cette formule. Il est en effet porté à craindre que des familles exploitent la force de travail des jeunes, ou qu'elles les privent afin que l'allocation versée pour leur entretien devienne une source de revenus; il se préoccupe aussi des conflits d'allégeance qui peuvent habiter des enfants pris entre parents naturels et parents nourriciers et des perturbations consécutives aux placements multiples que d'autres doivent subir ${ }^{27}$.

L'essentiel de son effort, pendant toutes ces années, est donc consacré à transformer les institutions trifluviennes dans le but d'en faire un milieu susceptible d'assurer aussi bien que possible le développement des enfants qui y sont placés. Ce qu'il faut pour eux tous, explique-t-il au journal Le Devoir en 1957, c'est y recréer «la dynamique du groupe familial ${ }^{28}$ ».

Sixième congrès - 1958, Compte rendu des conférences (Montréal, Secrétariat national, 1958), 112-125 et 128. On remarque la même chose en France: Pascale Quincy-Lefebvre, Familles, institutions et déviances. Une histoire de l'enfance difficile, 1880-fin des années 1930 (Paris, Économica, 1997), 367374.

27. Rivard exprime ouvertement ses craintes à de nombreuses occasions. Projet de rapport d'une journée d'étude des représentants des service sociaux faisant du placement en foyers nourriciers, tenue par le Comité spécial Foyers nourriciers de la commission «Enfance» de Caritas-Canada, Pavillon Saint-Arnaud, 2 mai 1958, dact., 13 p. (B. 1, ch. "Foyers nourriciers - Standards professionnels»); R. Rivard, ptre, Les services auxiliaires nécessaires au développement équilibré des enfants privés de famille normale, communication au $1^{\text {er }}$ congrès du CQEE, Montréal, 31 octobre, $1^{\text {er }}$ et 2 novembre 1963 , dact., 5 p. (B. 8, ch. «Conseil du Québec de l'enfance exceptionnelle»); «Jusqu’à 16 ans j’ai été un esclave", Le Foyer rural (avril 1955): 6 (témoignage d'un enfant placé) (B. 4, ch. "Enfants privés de famille - Institutions ou foyers nourriciers »); Ministère du Bien-être social, Service de l'enseignement, Rapport d'enquêtes sur quelques foyers nourriciers, 7 novembre 1960, 6 p. (B. 1, ch. "Normes professionnelles - Foyers nourriciers»). Les craintes de Rivard n'étaient pas complètement infondées. Voir Rose Dufour, Naître rien. Des orphelins de Duplessis: de la crèche à l'asile (Sainte-Foy, Éditions MultiMondes, 2002), 324 p.

28. «Le problème des enfants sans foyer. Le parfait équilibre de l'enfant dépend directement d'une dynamique familiale», Entrevue de Mario Cardinal avec l'abbé Reynald Rivard, d. ph., Le Devoir, 12 octobre 1957; "L'orphelinat... un donjon ou une cellule d'amour et de joie», Le Devoir, 19 octobre 1957. 


\subsection{La réforme des orphelinats}

Grâce aux démarches constantes de l'abbé puis $\mathrm{M}^{\mathrm{gr}}$ Bourgeois auprès des gouvernements et de donateurs privés, Reynald Rivard obtient les moyens financiers d'appliquer dans les orphelinats des Dominicaines du Rosaire, au tournant des années 1950, un programme complet de réformes visant à imprimer à ces grandes maisons pour enfants délaissés le caractère sinon du foyer familial, au moins d'un foyer de groupe ${ }^{29}$.

À l'instar de dom Bosco, il souhaite que la joie tienne un rôle de premier plan dans la vie des jeunes et que cela se manifeste jusque dans le nom des orphelinats. Dès 1948, il convainc les sœurs de changer ceux des leurs: Ville-Joie Saint-Dominique, pour les garçons de 6 à 12 ans, et Ville-Joie du Rosaire, pour les petits ${ }^{30}$.

L'espace est aussi réorganisé entièrement. Les murs et le mobilier sont peints de couleurs claires. Chaque groupe vit désormais dans des locaux bien à lui et d'ailleurs la taille de ces groupes diminue pour se rapprocher au plus près, compte tenu des coûts, des standards idéaux de l'époque. Les sœurs de la Providence délaissant progressivement leur œuvre des orphelines, les Dominicaines forment des groupes mixtes à Ville-Joie du Rosaire. C’est une nouveauté absolue dans le Québec des années 1950, mais une tendance encouragée en Suisse et aux Pays-Bas ${ }^{31}$. Entre 1945 et 1960, Rivard accumule d'ailleurs la documentation commerciale, gouvernementale et internationale sur l'organisation des garderies et des classes maternelles, sur les équipements qui leur conviennent et sur la formation des éducatrices ${ }^{32}$. Un gymnase, une piste d'hébertisme et une piscine intérieure entrent à Ville-Joie Saint-Dominique ${ }^{33}$.

29. L. Ferretti, Histoire des Dominicaines de Trois-Rivières. "C'est à moi que vous l'avez fait " (Sillery, Septentrion, 2002), 116-122.

30. ADT (Montréal), cahier 408. Projet “Ville-Joie», 11 mars 1948, dact., 6 p. À Sherbrooke, l'abbé Euchariste Paulhus suivra cet exemple. L'institut qu'il dirige prendra le nom de Centre Notre-Dame de la Joie.

31. B. 3, ch. "Enfants privés de famille. Colloque de Londres (9 au 18 juin 1952)». Communications de mademoiselle Hanni Waeber (Suisse) et de la docteure Schouten (Hollande) au colloque de Londres, 1952. Rivard s'est énormément inspiré de leurs propos dans sa réorganisation des orphelinats des dominicaines. Et aussi des réflexions de l'autre représentant du BICE à ce congrès: Claude Kohler, "Rééducation collective ou communautaire de l'enfant inadapté", Économie et humanisme, 71 (1952): 1-9 (B. 8, ch. «Projet de cours pour éducateurs spécialisés»). Voir Gérard Pelletier, Histoire des enfants tristes. Un reportage sur l'enfance sans soutien dans la province de Québec (Montréal, L’Action nationale, 1950), 95 p. L’orphelinat décrit comme progressiste par Pelletier est Ville-Joie Saint-Dominique.

32. B. 1, ch. "Classes maternelles».

33. "À Ville-Joie Saint-Dominique, étude des loisirs aux Journées sociales», Le Nouvelliste, 11 août 1950 . 
Parallèlement, on revoit l'éducation. Car, à la fin des années 1940, les religieuses et les autres enseignantes évaluent que sur 350 enfants de VilleJoie Saint-Dominique, une cinquantaine ne peuvent suivre le cours régulier correspondant à leur âge. En 1951, les premières classes spéciales du diocèse sont donc organisées dans les deux orphelinats des Dominicaines ainsi qu'au Patronage Saint-Charles. Les unes sont dites de "récupération» et les autres, pour les "caractériels ${ }^{34}$ ». Dès lors, l’abbé Rivard ne cessera plus de s'intéresser à tout ce qui touche l'éducation des jeunes déficients et handicapés, où que ce soit au monde: l'organisation des institutions, la formation des éducateurs, les soins à donner, les formules pédagogiques, le matériel, etc. ${ }^{35}$.

Ces classes s'adressent à des enfants dotés d'un quotient intellectuel de 50 à 80 , c'est-à-dire classés au-dessus des «imbéciles» mais sous les «lents intellectuellement». Elles proposent un programme comprenant une formation scolaire élémentaire, et surtout une formation sociale et occupationnelle dont l'objectif est de rendre les jeunes aussi indépendants que possible ${ }^{36}$. On vise certes la progression de leur intelligence, mais surtout le développement de leurs compétences sociales, de leur capacité d'adaptation à la vie en société. Au Patronage, on leur donne aussi un contact avec le milieu du travail. Les professeurs spécialisés laïques et religieux enseignent à l'aide d'un matériel didactique qui fait une large part à la musique et surtout aux moyens audiovisuels. Un psychologue pour la rééducation ainsi qu'un éducateur spécialisé chargé de l'éducation psychomotrice sont embauchés en 1955. Enfin, les anciens du Patronage continuent d'être suivis par un travailleur social qui les conseille et qui les aide. Les rapports annuels de Clément Thibert, responsable de l'éducation spécialisée à l'Institut psycho-social, permettent toutefois de constater sans surprise que les résultats les meilleurs sont atteints chez les plus jeunes ${ }^{37}$.

34. R. Rivard, Mémoire présenté au Conseil des Révérendes Sœurs Dominicaines du Rosaire concernant le problème des arriérés mentaux à Ville-Joie Saint-Dominique, 2 octobre 1951, dact., 3 p. (B. 7, ch. «Classes spécialisées»). En 1951, le Patronage Saint-Charles est vendu par les Dominicaines du Rosaire aux frères de Saint-Vincent-de-Paul.

35. Les b. 7 et 8 , surtout, sont pleines de documents qui témoignent de l'intérêt durable et profond de Rivard pour tous ces sujets; on en trouve d'autres dans les b. 3 et 18. À noter, l'abondance de la documentation provenant des États-Unis.

36. Clément Thibert, "Nos œuvres... Classes spéciales pour déficients mentaux», La vie des œuvres, II,4 (décembre 1956); et Évaluation du programme des classes spécialisées à Ville-Joie SaintDominique et au Patronage Saint-Charles, par le directeur technique de l'IPS (c.-à-d. R. Rivard), 9 juin 1955, dact., 3 p. (B. 7, ch. "Classes spécialisées»).

37. R. Rivard, Essai de solution du problème des sous-doués dans les institutions pour enfants privés de famille aux Trois-Rivières, Préparation d'une conférence pour la Journée d'étude du Conseil des 
Par ailleurs, les Dominicaines acceptent de repenser entièrement l'organisation de la vie des jeunes en dehors des heures de classe. L'objectif : favoriser leur stabilité émotive, condition, selon l'abbé Rivard, de leur développement dans tous les domaines, y compris intellectuel.

Afin d'amoindrir le sentiment des jeunes d'être rejetés par leur famille, on cherche désormais à mieux maintenir leurs liens avec leurs parents, leurs frères et sœurs restés au foyer et ceux qui sont placés ailleurs; dans ce but, les religieuses invitent à l'occasion les familles dans leurs orphelinats et encouragent aussi les parents à venir chercher leurs jeunes pour des sorties ${ }^{38}$; elles jumellent avec des familles amies ceux qui sont sans parents ni parenté ${ }^{39}$. Pour faire expérimenter le succès aux enfants, source d'épanouissement et de joie, un véritable programme d'activités parascolaires inclut des échanges sportifs entre les orphelins et les élèves des autres écoles ainsi que du théâtre, de la danse, des cours de diction, des sorties culturelles, des séances d'observation de la nature et des oiseaux ainsi que du bricolage ${ }^{40}$.

Des spécialistes sont embauchés pour les arts et pour la gymnastique; car à l'Université d'Ottawa, Rivard a été sensibilisé à l'apport de l'éducation physique au développement intégral de la personnalité et il croit à la nécessité d'une présence masculine auprès des garçons ${ }^{41}$. Par ailleurs, les

œuvres à Québec, 25 janvier 1957, et Rapport annuel sur l'évolution de l'enseignement spécialisé (sousdoués) au Patronage Saint-Charles, 1958-1959, par Clément Thibert, psychologue consultant, conseiller technique en éducation spéciale, 22 juin 1959, dact., 3 p. (B. 7, ch. «Classes spécialisées ").

38. Rapport de la Commission II de la session d'étude tenue sous les auspices de la Commission française du Conseil canadien du bien-être social, voir note 26.

39. Rapport du Centre de service social du diocèse de Trois-Rivières, 1953, 27, et Ibid., 1955, 9. (Ces rapports sont disponibles à la bibliothèque de l'UQTR); ADT (Trois-Rivières), Régina Doyon, o.p., Bref historique des œuvres des Dominicaines du Rosaire, Trois-Rivières, manuscrit; ADT (Montréal), cahier 399. Chronique de Ville-Joie Saint-Dominique, 28 avril 1954, 5 février et 29 mars 1956; et Délibérations du conseil général, 13 juin 1955 et 23 avril 1959.

40. Plusieurs documents sur le jeu et les jeux sont conservés dans la b. 5, et dans la b. 10, ch. "Récréations - Trousse d'étude». L’idée de favoriser la socialisation par les loisirs est aussi très présente à l'OTJ par exemple. Elle prend de l'ampleur en ces années des deux côtés de l'Atlantique. Pour quelques exemples français: Dominique Dessertine et Bernard Marandan, L'âge d'or des patronages (1919-1939). La socialisation de l'enfance par les loisirs (Vaucresson, Ministère de la Justice, CNFE-PJJ, 2001), 235 p. ; Laura Lee Downs, Childhood in the Promised Land: Working-Class Movements and the Colonies de Vacances in France, 1880-1960 (Durham, Duke University Press, 2002), xv-411 p.

41. Antoine Paplauskas-Ramunas, L'éducation physique dans l'humanisme intégral (Ottawa, Les Éditions de l'Université d'Ottawa, 1954), 115 p., première édition; Comité d'études sur la formation des éducateurs de l'enfance exceptionnelle, Compte rendu de la réunion tenue au Centre NotreDame de la Joie, à Rock Forest, près Sherbrooke, vendredi 27 mai 1960, à 10 h, dact., 5 p. (B. 8, ch. «Éducation de l'enfance exceptionnelle. Formation des éducateurs»). 
groupes sont divisés en équipes de 6 ou 7 enfants qui, comme les scouts, développent les uns vis-à-vis des autres un sentiment de responsabilité personnelle et collective devant les tâches qui leur sont confiées. Les sœurs multiplient aussi les mesures pour favoriser le développement de la personnalité individuelle: cela passe entre autres par l'abandon du costume remplacé par la confection de trousseaux personnels, l'octroi de temps, de jeux et de casiers bien à soi.

Certes, les religieuses et l'abbé Rivard savent que ces réformes ne viennent pas à bout de tous les retards de développement ni de la souffrance des orphelins les plus abandonnés. Cependant, elles améliorent la qualité de vie et d'éducation des enfants placés d'une façon telle que le prêtre psychologue peut estimer qu'une institution repensée de cette manière vaut bien le foyer nourricier.

Mais encore faut-il que les religieuses soient tenues à jour des derniers développements du savoir sur l'enfance et bénéficient d'une formation appropriée.

\section{3 - PSYCHOLOGIE, PSYCHOPÉDAGOGIE ET HABILETÉS TECHNIQUES: LA FORMATION D'ÉDUCATRICES SPÉCIALISÉES}

Car parallèlement et bien dans l'esprit de l'époque, qui est à la professionnalisation du travail auprès des enfants et des familles, l'abbé Rivard entreprend de mieux former les religieuses. Comme le dit l'abbé Henri Bissonnier, secrétaire général de la Commission médico-sociale et psychopédagogique du BICE, le prêtre psychologue veut rendre celles-ci capables de "concilier dévouement et charité avec compétence et technicité ${ }^{42}$ ». Perfectionnement, formation continue, ces mots ne sont pas encore employés, mais c'est bien dans un tel processus que s'engagent alors les religieuses.

Les premières Journées sociales à Ville-Joie Saint-Dominique, en 1949, inaugurent une formule qui va durer $10 \mathrm{ans}^{43}$. Toutes les éducatrices des orphelinats du diocèse de Trois-Rivières, de toutes les congrégations, y sont invitées: l'assistance tourne autour de deux cents religieuses dans les premières années ${ }^{44}$. Pendant cinq jours, au mois d’août, celles-ci sont dégagées

42. Lettre de Henri Bissonnier de la Commission médico-légale et psycho-pédagogique du BICE à Reynald Rivard, Paris, 25 janvier 1955 (B. 7, ch. «Enfance déficiente»). L'abbé Bissonnier est une référence internationale dans le milieu de l'éducation spécialisée à cette époque.

43. B. 16 , ch. "Cours de perfectionnement aux religieuses, Ville-Joie Saint-Dominique» : il y en a une pour chacune des dix années d'existence de ces cours.

44. L'assistance tourne autour d'une centaine, parfois moins, à partir de 1954; il faut dire que l'abbé Rivard a alors commencé à offrir des sessions pour d'autres groupes de religieuses. 
de leur emploi pour suivre un programme de conférences prononcées par plusieurs spécialistes et complété peu à peu par des films éducatifs, une exposition de matériel pédagogique et de livres, et, à partir de 1955, par des ateliers au cours desquels les participantes discutent des problèmes d'éducation qu'elles rencontrent et des manières de les résoudre.

En 1951 commence la collaboration avec Roger Dion, directeur adjoint de l'Institut d'éducation physique et de récréation de l'Université d'Ottawa; des cours sur les techniques audiovisuelles s'ajoutent en 1953, la dynamique des groupes en 1956. Les thèmes varient d'une année à l'autre: hygiène infantile, croissance et développement de l'enfant, programmation rationnelle des loisirs, techniques de gymnastique, psychologie, techniques d'éducation religieuse et usage raisonné des exercices de piété, causes et traitement de l'énurésie, responsabilités de chaque membre du personnel dans l'éducation des jeunes. On le voit: les exposés sur la doctrine cèdent carrément le pas aux questions concrètes.

Les sœurs sont encouragées à s'exprimer sur tout sujet qui les préoccupe. Comment éviter de séparer des frères d'âges très différents dès leur entrée à l'orphelinat; la directrice peut-elle dire à la religieuse qui s'occupe d'un enfant que celui-ci est illégitime? Que faire du garçon impoli avec une sœur devant ses compagnons (1949)? Non, ce n’est pas péché de faire prendre le bain sans jupette aux enfants, ni que ceux-ci «se touchent». Quelles mesures prendre pour arrêter celui qui dissipe tout le monde au réfectoire ou au dortoir sans l'empêcher tout de même d'évacuer son trop-plein d'énergie? Est-ce un grave manquement à la charité si un jeune de 13 ou 14 ans parle contre ses parents, les mettant même au rang des animaux? Pour ne pas dégoûter les enfants de la religion, doit-on faire de la messe une option sauf le dimanche et les entraîner à y participer plus activement par le dialogue ou du chant (1950)? Comment limiter la consommation de friandises tout en sauvant la valeur psychologique du sac de bonbons apporté par les parents? Encourageons l'initiative chez nos enfants en leur laissant organiser eux-mêmes, avec une discrète assistance, des veillées récréatives. Quand on connaît la facilité des orphelins à s'attacher à quiconque leur tend la main, comment s'assurer qu'ils ne soient pas déçus si les marraines religieuses ou les familles amies à qui on les jumelle cessent un jour de les visiter ou de les sortir (1955)?

Bref, l'abbé Rivard cherche à rassurer et à outiller les religieuses dans toutes les situations de la vie quotidienne avec des enfants internés. Il les sensibilise aussi à l'idée d'accueillir un travailleur social dans l'institution même. 
Bientôt, sa réputation s'étend. À la demande des Sœurs de la Providence, il consacre une semaine intensive chaque été, de 1953 à 1958, à des cours plutôt théoriques sur la psychologie de l'enfance, qui rejoignent au total 340 religieuses de cette congrégation ${ }^{45}$. Mais surtout, l'Association patronale des services hospitaliers, qui regroupe depuis 1944 les administratrices et administrateurs d'hôpitaux et d'établissements d'hébergement catholiques, le requiert chaque année, entre 1952 et 1958, pour des sessions de 2 puis 3 jours données à la maison Notre-Dame du Cénacle, à Québec. Y assistent environ 200 sœurs éducatrices de divers orphelinats ordinaires situés un peu partout au Québec, dans une formule qui ressemble beaucoup à ce qui se fait à Ville-Joie Saint-Dominique. Peu à peu, devant les aspirations des religieuses à la formation professionnelle, et compte tenu que la sous-commission «Institution» de la commission «Enfance» de Caritas-Canada demande périodiquement la structuration de ces leçons, germe l'idée de bâtir un programme de formation qui conduirait à l'obtention d'un brevet de compétence ${ }^{46}$.

En cette matière comme dans les autres, Rivard se montre très soucieux de ce qui se fait ailleurs. Au cours des années 1950, à la demande de l'UNESCO, le BICE entreprend une vaste enquête internationale sur la formation professionnelle des éducatrices spécialisées. Son bulletin mensuel, L'enfance dans le monde, fait part d'expériences conduites surtout en Europe ainsi que du programme-type que l'organisme propose et suggère d'adapter aux situations particulières ${ }^{47}$. Rivard y cherche son inspiration, qu'il trouve aussi aux États-Unis, notamment à l'École de service social de l'Université Saint-Louis, et en Angleterre.

Tout cela le conduit à concevoir un programme dit de "mères de groupe ", en collaboration avec l'Institut d'éducation physique, d'hygiène et de récréation de l'Université d'Ottawa. Il s'agit d'un programme de formation continue à l'intention des religieuses qui s'occupent des enfants

45. B. 15, dossier Cours de psychologie aux sœurs de la Providence, 1953-1958.

46. Plusieurs mentions dans divers documents conservés dans B. 16, ch. "Cours de psychopédagogie aux sœurs St-Joseph de St-Vallier - 1952-1958». R. Rivard, Formation des éducatrices spécialisées dans le soin des enfants privés de famille, Trois-Rivières, février 1960, dact., 3 p. (B. 8, ch. "Projet de cours pour éducateurs spécialisés. Caritas-Canada, 1960-1961»).

47. B. 1, ch. "Classes maternelles». Plusieurs articles détaillés sur des programmes de formation conçus à Anvers, Lyon, Paris dans L'enfance dans le monde, 2,2 (15 février 1954): 24-30; et Ibid., 37 (août-septembre 1955): 28-30; R. Rivard, Formation des éducatrices..., op. cit.. ; il se réfère aussi au livre de Eva Burneister, The Professional House Parent (New York, Columbia University Press, 1960), 244 p. (B. 8, ch. "Projet de cours pour éducateurs spécialisés. Caritas-Canada, 1960-1961»). Ne sont pas notées ici des sources d'inspiration moins importantes, mais dont on retrouve des traces dans le fonds Rivard. 
des orphelinats en dehors des heures de classe ${ }^{48}$. Au moment de l'ouverture de l'École des «mères de groupe», en 1959, 12000 jeunes sont hébergés dans les orphelinats ordinaires du Québec; 10000 en 1960. En comptant ne serait-ce qu'une seule éducatrice par groupe de 25 enfants, Rivard calcule qu'il faudrait donc en former plus de 400. Le tableau I résume les caractéristiques du cours, enseigné par 7 professeurs la première année, par entre 10 et 15 ensuite. À raison d'un mois par année, durant l'été, il s'étale sur 3 ans, puis 4 à partir de 1964. Les sœurs de diverses congrégations quittent alors leur couvent pour vivre toutes ensemble au camp que possède l'Université à Gracefield dans la Gatineau. La formation est complétée par un stage, dont la réussite est conditionnelle à l'obtention du diplôme. Le nombre d'inscriptions, 18 en 1959, augmente ensuite d'année en année à mesure que de nouvelles cohortes sont constituées ${ }^{49}$.

La formation poursuit les trois mêmes objectifs que les Journées sociales. Tout d'abord, fournir aux religieuses un savoir de base en psychologie de l'enfance (115 heures en 1959, 120 ensuite). Mais désormais, en plus d'enseigner les notions fondamentales, les facteurs de développement, l'hygiène mentale et les problèmes émotifs, Reynald Rivard les initie à la dynamique de groupe, en leur apprenant à reconnaitre les forces inhérentes d'un groupe et comment les utiliser. Deuxième objectif : faire acquérir aux sœurs des méthodes de travail appropriées; cela signifie l'étude en équipe des cas individuels, la participation à des rencontres régulières entre membres du personnel, une meilleure connaissance du rôle et de la personnalité de l'éducatrice spécialisée, la manière de créer un climat favorable dans l'institution, d'organiser les récréations et de monter des programmes d'activités et de sorties; 115 heures en 1959, 100 ensuite sont donc consacrées à ces divers volets de la formation en psychopédagogie, destinée à assurer plus d’unité de pensée et d'action dans l'institution, donc plus de stabilité émotive chez les enfants. Enfin, troisième objectif : la maîtrise de diverses techniques de jeux, incluant l'édu-

48. B. 17, ch. «Cours pour éducatrices des orphelinats, Université d'Ottawa, 1959-1964». Document sans titre [Horaire des cours], s.d. [1959], dact., 1 p. (B. 8, ch. «Projet de cours pour éducateurs spécialisés"); L'École des Sciences sociales de Trois-Rivières en collaboration avec l'Institut d'éducation physique, d'hygiène et de récréation de l'Université d'Ottawa offre un cours d'Éducation spéciale pour les religieuses en charge des enfants privés de famille normale, s.d. [1960], dact., 2 p. (B. 8, ch. "Éducation de l'enfance exceptionnelle. Formation des éducateurs»); ADT (Trois-Rivières), Archives personnelles de sr Bernadette Lavergne, o.p., Cours de Mère de groupe, 4 cahiers, 1964 à 1967.

49. Brochure souvenir Cours Mères de groupe. 10 ans 1958-1968 (B. 16, ch. jaune). 
cation physique et l'expression corporelle, les travaux manuels comme le bricolage et le dessin, la musique et la danse, le théâtre, le mime, les marionnettes, l'ornithologie, la connaissance de la nature et enfin les techniques audiovisuelles; le tout sur 345 heures pour la cohorte de 1959, réduites à 250 pour les suivantes ${ }^{50}$. On parle donc d'une formation de 470 heures complétées par un stage.

L'abbé Rivard voit plusieurs avantages à ces sessions intensives près d'un lac, loin du cadre de vie habituel. Plus détendues, les religieuses sont davantage concentrées. Elles apprennent à se connaître entre congrégations, se transmettent les façons de faire dans la leur. Et surtout, elles bénéficient elles-mêmes de toutes les techniques qu'elles apprennent en vue d'aider les enfants à s'exprimer. Les cours de «mères de groupe » sont l'occasion de libérer leur propre personnalité, de la laisser poindre dans les nombreuses activités artistiques qu'elles expérimentent, d'oser parler dans les ateliers et réfléchir à haute voix, de découvrir leurs talents et d'en apprendre sur elles-mêmes, de vivre en confiance dans le groupe et de se faire confiance à elles-mêmes. Ce n'est pas la moindre retombée de cette formation ${ }^{51}$.

Mais au moment même où le prêtre psychologue couronne par la mise sur pied du cours de "mères de groupe» la réforme qu'il a entreprise des orphelinats ordinaires, au moment où il peut penser que ces maisons ainsi modernisées et équipées et ces religieuses mieux outillées offrent un havre valable aux enfants sans foyer, les changements en cours dans la culture globale, dans la société et dans le milieu des professionnels de l'enfance exceptionnelle dessinent les contours d'un nouveau contexte.

\section{4 - LES ANNÉES 1960: LA NOUVELLE CONFIGURATION DE L'ÉDUCATION SPÉCIALISÉE}

Au tournant des années 1960, le système de prise en charge des enfants exceptionnels est en transition, il est en voie de reconfiguration sous l'effet de plusieurs facteurs: des changements dans la culture globale, le développement des savoirs relatifs à l'enfance, mais aussi les rapports de force entre les différents groupes d'acteurs qui y sont impliqués.

50. Pour le contenu leçon par leçon: Cours d'éducation spéciale pour les religieuses en charge des enfants privés de famille normale, dact., 3 p. (B. 8, ch. "Projet de cours pour éducateurs spécialisés - Caritas-Canada, 1960-1961»).

51. Comité d'études sur la formation des éducateurs de l'enfance exceptionnelle, Compte rendu de la réunion..., vendredi 27 mai 1960, voir note 41; témoignage recueilli auprès de madame Anita Cadotte à l'automne 2008; nombreux témoignages de mères de groupe dominicaines reçus au fil des ans par l'auteure. 


\section{I La dévalorisation de la formation de mères de groupe}

Les années 1950 ont été celles de l'expansion et de la diversification des établissements d'hébergement ainsi que d'une véritable recherche quant aux connaissances et aux habiletés nécessaires aux hommes et aux femmes chargés d'accompagner les enfants handicapés ou mésadaptés dans leur vie quotidienne en institution. Si bien qu'à la fin de la décennie, en plus de ce qui s'enseigne dans les Écoles de service social pour préparer les travailleurs sociaux de groupe et des programmes de l'Université McGill, 4 types de formation différentes existent à l'intention des éducateurs de l'enfance exceptionnelle à part celle des mères de groupe $\mathrm{e}^{52}$.

La consultation du tableau 1 montre bien la variété de ces formations. Dans l'espoir d'en arriver à une meilleure coordination, le service des études du ministère du Bien-être social et la commission «Institution » de Caritas-Canada forment chacun de son côté un comité chargé d'étudier la question. Comme les autres responsables des formations offertes, Reynald Rivard est invité à siéger à tous deux ${ }^{53}$.

Les réunions se succèdent pendant des mois sans qu'on réussisse à s'entendre. La tendance serait à imposer à tous les étudiants le préalable de la $11^{\mathrm{e}}$ année, à encourager la formation de niveau universitaire, à préférer la formation initiale à la formation continue, et à attirer des jeunes hommes dans la profession. Bref, à qualifier les étudiants en vue de les faire reconnaître comme de véritables professionnels, à côté des autres, tels les travailleurs sociaux par exemple, qui ont investi de plus en plus sûrement depuis les années 1950 le monde de l'enfance exceptionnelle. C'est la position défendue entre autres par le Centre d'orientation et l'Université de Sherbrooke. L'abbé Rivard fait valoir ses arguments: manque de préparation de plusieurs institutions ; religieuses souvent plus expérimentées que diplômées; nécessité d'adapter la formation en fonction des catégories d'enfants à éduquer; et, en formation continue, supériorité de la session intensive d'été sur celle des leçons à temps partiel offertes toute l'année. À la fin, le comité de Caritas-Canada décide de maintenir le cours de "mères de groupe» pour les religieuses; et de monter, parallèlement, un nouveau programme à l'intention des éduca-

52. Lettre de Louis-Philippe Audet, directeur des études au ministère du Bien-être social aux membres du Comité d'études sur l'enfance exceptionnelle, Montréal 14 mars 1960 plus 2 annexes (B. 8, ch. "Éducation de l'enfance exceptionnelle. Formation des éducateurs»). Une autre option de ces programmes prépare à l'enseignement aux déficients.

53. B. 8, ch. «Projet de cours pour éducateurs spécialisés. Caritas-Canada, 1960-1961»; B. 8, ch. «Éducation de l'enfance exceptionnelle. Formation des éducateurs». 
Tableau I

Caractéristiques des programmes de formation pour les éducateurs de l'enfance exceptionnelle à la fin des années 1950

\begin{tabular}{|c|c|c|c|c|c|}
\hline & $\begin{array}{l}\text { Cours de } \\
\text { mères de } \\
\text { groupe } \\
\text { Depuis } 1959^{1}\end{array}$ & $\begin{array}{l}\text { Formation } \\
\text { en psycho- } \\
\text { pédagogie } \\
\text { Depuis } 1954^{2}\end{array}$ & $\begin{array}{c}\text { Formation } \\
\text { en pédagogie } \\
\text { Depuis } 1958^{3}\end{array}$ & $\begin{array}{l}\text { Formation } \\
\text { en éducation } \\
\text { spécialisée } \\
\text { Depuis } 1959^{4}\end{array}$ & $\begin{array}{c}\text { Cours } \\
\text { de socio-pédagogie } \\
\text { Depuis } 1959^{5}\end{array}$ \\
\hline Institution & $\begin{array}{l}\text { Rivard + } \\
\text { Institut } \\
\text { d'éducation } \\
\text { physique, } \\
\text { d'hygiène et de } \\
\text { récréation de } \\
\text { l'Université } \\
\text { d'Ottawa }\end{array}$ & $\begin{array}{l}\text { Centre } \\
\text { d'orientation } \\
\text { de l'U. de } \\
\text { Montréal et } \\
\text { U. de } \\
\text { Sherbrooke }\end{array}$ & $\begin{array}{l}\text { École de } \\
\text { pédagogie et } \\
\text { d'orientation } \\
\text { de l'U. Laval }\end{array}$ & $\begin{array}{l}\text { École } \\
\text { normale } \\
\text { Jacques- } \\
\text { Cartier }\end{array}$ & $\begin{array}{l}\text { Centre de socio-pédagogie du } \\
\text { ministère du Bien-être social, } \\
\text { affilié au DIP et considéré } \\
\text { comme une école normale }\end{array}$ \\
\hline $\begin{array}{l}\text { Type } \\
\text { d'étudiants } \\
\text { visés } \\
\text { prioritaire- } \\
\text { ment ou } \\
\text { exclusive- } \\
\text { ment }\end{array}$ & Les religieuses & $\begin{array}{l}\text { Jeunes } \\
\text { hommes } \\
\text { laïques }\end{array}$ & $\begin{array}{l}\text { Le personnel } \\
\text { des institu- } \\
\text { tions pour } \\
\text { déficients } \\
\text { mentaux }\end{array}$ & $\begin{array}{l}\text { Hommes, } \\
\text { laïques et } \\
\text { religieux }\end{array}$ & $\begin{array}{l}\text { Hommes: éducateurs, } \\
\text { moniteurs, officiers de } \\
\text { probation, officiers enquêteurs } \\
\text { déjà à l'œuvre dans les écoles } \\
\text { de protection. }\end{array}$ \\
\hline $\begin{array}{l}\text { Formation } \\
\text { préalable } \\
\text { exigée }\end{array}$ & $\begin{array}{l}\text { Idéalement, } 11^{\mathrm{e}} \\
\text { ou } 12^{\mathrm{e}} \text { année. } \\
\text { Dans les faits, } \\
\text { on accepte sans } \\
\text { considérer la } \\
\text { scolarité }\end{array}$ & $12^{\mathrm{e}}$ année & $12^{\mathrm{e}}$ année & $11^{\mathrm{e}}$ année & $\begin{array}{l}11^{\mathrm{e}} \text { année. } \\
\text { Dans les faits, la formation } \\
\text { préalable des étudiants est } \\
\text { supérieure. }\end{array}$ \\
\hline Forme & $\begin{array}{l}575 \text { heures de } \\
1959 \text { à } 1963 ; \\
470 \text { heures à } \\
\text { partir de } 1964 \\
+ \text { stage } \\
\text { supervisé d'un } \\
\text { an. }\end{array}$ & $\begin{array}{l}2 \text { ans à temps } \\
\text { partiel } \\
\text { (incluant } \\
\text { études } \\
\text { théoriques en } \\
\text { psychologie } \\
\text { et en } \\
\text { pédagogie + } \\
\text { stage) }\end{array}$ & & 1 an & $\begin{array}{l}495 \text { heures sur } 4 \text { ans, } \\
\text { équivalent à } 1 \text { an à temps } \\
\text { complet } \\
\text { (formation théorique et } \\
\text { pratique complétée par un } \\
\text { stage) }\end{array}$ \\
\hline $\begin{array}{l}\text { Diplôme } \\
\text { obtenu }\end{array}$ & $\begin{array}{l}\text { Diplôme de } \\
\text { Mères de } \\
\text { groupe }\end{array}$ & $\begin{array}{l}\text { Certificat en } \\
\text { psychopéda- } \\
\text { gogie pour } \\
\text { l'enfance } \\
\text { inadaptée }\end{array}$ & $\begin{array}{l}\text { Baccalauréat } \\
\text { en pédagogie } \\
\text { option } \\
\text { «mésadaptés» } \\
\text { équivalent au } \\
\text { brevet de } \\
\text { classe A } \\
\text { Ou } \\
\text { Brevet } \\
\text { spécialisé } \\
\text { pour les } \\
\text { enfants } \\
\text { mésadaptés, } \\
\text { de classe B. }\end{array}$ & $\begin{array}{l}\text { «Brevet } \\
\text { spécial» }\end{array}$ & $\begin{array}{l}\text { Brevet d'éducateur de l'enfance } \\
\text { exceptionnelle décerné par le } \\
\text { DIP }\end{array}$ \\
\hline
\end{tabular}




\begin{tabular}{|c|c|c|c|c|c|}
\hline & $\begin{array}{c}\text { Cours de } \\
\text { mères de } \\
\text { groupe } \\
\text { Depuis } 1959^{1}\end{array}$ & $\begin{array}{l}\text { Formation } \\
\text { en psycho- } \\
\text { pédagogie } \\
\text { Depuis } 1954^{2}\end{array}$ & $\begin{array}{c}\text { Formation } \\
\text { en pédagogie } \\
\text { Depuis } 1958^{3}\end{array}$ & $\begin{array}{c}\text { Formation } \\
\text { en éducation } \\
\text { spécialisée } \\
\text { Depuis } 1959^{4}\end{array}$ & $\begin{array}{c}\text { Cours } \\
\text { de socio-pédagogie } \\
\text { Depuis } 1959^{5}\end{array}$ \\
\hline Titre & Mère de groupe & $\begin{array}{l}\text { Éducateur } \\
\text { spécialisé } \\
\text { pour jeunes } \\
\text { inadaptés }\end{array}$ & & Éducateurs & $\begin{array}{l}\text { Éducateurs de l'enfance } \\
\text { exceptionnelle }\end{array}$ \\
\hline $\begin{array}{l}\text { Lieux de } \\
\text { pratique }\end{array}$ & $\begin{array}{l}\text { Orphelinats } \\
\text { ordinaires }\end{array}$ & $\begin{array}{l}\text { Entre autres à } \\
\text { Boscoville et } \\
\text { au Centre } \\
\text { d'orientation } \\
\text { de l'U. de } \\
\text { Montréal }\end{array}$ & $\begin{array}{l}\text { Les } \\
\text { institutions } \\
\text { pour } \\
\text { déficients } \\
\text { mentaux }\end{array}$ & $\begin{array}{l}\text { Orphelinats } \\
\text { et écoles de } \\
\text { protection de } \\
\text { la jeunesse }\end{array}$ & $\begin{array}{l}\text { Écoles de protection de la } \\
\text { jeunesse }\end{array}$ \\
\hline
\end{tabular}

1. Comité d'études sur la formation des éducateurs de l'enfance exceptionnelle, Compte rendu de la réunion tenue au Centre Notre-Dame de la Joie, à Rock Forest, près Sherbrooke, vendredi 27 mai 1960, à 10 h, dact., 5 p. (B. 8, ch. «Éducation de l'enfance exceptionnelle. Formation des éducateurs»).. R. Rivard, «École de formation des mères de groupe», dans L'enfance exceptionnelle. Mémoire présenté... par le Conseil des aeuvres de Montréal, avril 1962, 339346.

2. Lettre de Gilles Gendreau, de l'Association des éducateurs spécialisés pour jeunes inadaptés à l'abbé R. Rivard, Rivières-des-Prairies, s.d. [autour de 1960] +1 annexe (B. 8, ch. «Projets de cours pour éducateurs spécialisés»). Cette formation a été conçue essentiellement par le père Noël Mailloux, o.p., par Jeannine Guindon et par l’abbé Euchariste Paulhus.

3. Université Laval, École de pédagogie et d'orientation, Cours en vue de l'enseignement aux déficients mentaux, 4 p. (B. 7, ch. «Enfance déficiente»); et Document sans titre sur les brevets d'enseignement, s.d., [autour de 1960], 2 p. (B. 8, ch. «Éducateurs de cadre»).

4. "Trois nouveaux cours créés pour enfants et adultes au Québec», La Presse, 22 décembre 1959.

5. Lettre de L.-P. Audet, dir. des centres de socio-pédagogie aux membres de la Commission des Écoles Normales, 19 oct. 1959 (B. 6, ch. «Nouveau programme des Écoles normales - Cours à l'étude»); Les Centres de socio-pédagogie. Cours de perfectionnement pour les éducateurs de l'enfance exceptionnelle, dact., 2 p. et Caritas-Canada, Commission «Enfance», sous-commission «Institutions», comité «Éducateur» (réunion spéciale), Projet de rapport de la réunion tenue au Centre de service social de Trois-Rivières le lundi 20 janv. 1961 (B. 8, ch. «Projet de cours pour éducateurs spécialisés. Caritas-Canada, 1960-1961»).

teurs, laïques et religieux, en orphelinat. Celui-ci exigera la $11^{\mathrm{e}}$ année, et on en demandera la reconnaissance au département de l'Instruction publique. En somme, il s'agira de la formule des centres de socio-pédagogie mais adaptée à des enfants mésadaptés plutôt que délinquants ${ }^{54}$.

Dès lors, on saisit bien que la formation de mères de groupe subit une première dévalorisation. Toutes les autres sont supérieures et conduisent à un «brevet spécial», à des brevets ou à des baccalauréats. Les jeunes hommes et les religieux qui les suivent travaillent certes surtout auprès des adolescents. Mais désormais, de plus en plus d'hommes laïques sont

54. R. Rivard, Formation des éducatrices spécialisées..., voir note 46; et Annexe à la Lettre de Louis-Philippe Latulippe, ptre, secrétaire général de Caritas-Canada, à l'abbé Reynald Rivard, Centre psycho-social de Trois-Rivières, Montréal, 20 janvier 1961 (B. 8, ch. «Projet de cours pour éducateurs spécialisés. Caritas-Canada, 1960-1961»). 
qualifiés pour accompagner même les enfants. Dans leur désir sincère de venir en aide à ceux-ci le mieux possible, les religieuses en charge des orphelinats ordinaires sont d'ailleurs heureuses d'embaucher de tels éducateurs quand cela leur est possible financièrement; d'autant plus que depuis quelques années, à mesure qu'augmente le scepticisme de la société sur leur propre capacité de préparer des jeunes à la vie adulte, les psychologues estiment plus nécessaire une présence masculine régulière dans les institutions. Ces jeunes professionnels et les religieux qui s'apprêtent à se rapprocher du ministère de la Famille et du Bien-être social ne tarderont pas à indiquer aux sœurs, même «mères de groupe », que c'est sur eux que repose désormais la crédibilité des institutions et qu’en fait, elles-mêmes y nuisent.

\subsection{Les travailleurs sociaux et la métamorphose des orphelinats ordinaires}

Car en effet, en ce début des années 1960, les orphelinats sont dans la mire de la société. Malgré les visibles améliorations apportées depuis l'enquête choc de Gérard Pelletier en 1950, les lacunes de ces établissements ne cessent plus d'être mises en lumière. Grâce à la prospérité de ces années, beaucoup de parents sont heureux d'éviter d'avoir à leur confier leurs enfants. Les mères célibataires, quant à elles, décident plus souvent qu’autrefois de garder leur bébé. Dans les agences et les centres de services sociaux enfin, les travailleurs sociaux favorisent toujours davantage le placement en foyer nourricier et ils ne recommandent à présent l'internement que pour les jeunes les plus atteints ${ }^{55}$. En 1958 déjà, sœur Suzanne Colette, directrice du Bureau d'admission à la Crèche d'Youville, a fait remarquer que les orphelinats accueillent désormais surtout des jeunes très marqués et provenant de foyers beaucoup plus problématiques qu'auparavant ${ }^{56}$. En 1961-1962, plus d'enfants vivent désormais en foyers

55. Avec la plupart des intervenants, les travailleurs sociaux sont convaincus de la supériorité du placement familial; ils sont aussi déçus de leur peu d'accès aux enfants vivant en institution. N. Bélanger, entre autres chercheurs, a pu montrer que les besoins des enfants étant définis par les professionnels, ceux-ci ont naturellement tendance à proposer, parmi les solutions possibles, celles qui tiennent le mieux compte de paramètres avantageux pour eux (De la psychologie scolaire à la politique de l'enfance inadaptée, op. cit., voir note 13). Or, l'idéal de l'époque est d'un travailleur social au moins dans chaque institution, mais de deux travailleurs sociaux pour surveiller chaque cas de placement en foyer nourricier (un pour suivre la famille et l'autre, l'enfant), avec une moyenne de 25 cas par travailleur social (R. Rivard et G. Lacroix, Essai de normes professionnelles..., op. cit., voir note 21).

56. Sr Suzanne Cholette, s.g.m., «La réception de l'enfant: les ressources de l'institution», Caritas-Canada, "La charité dans la charité», Sixième congrès - 1958, op. cit., 131-135. 
nourriciers (16 330) qu'en institution (14 936) $)^{57}$, une tendance qui va en s'accentuant. Des établissements ferment leurs portes: en 1960, il y a 86 orphelinats ordinaires au Québec, 75 en $1962^{58}$.

D’autres enjeux apparaissent. À Trois-Rivières comme ailleurs, les centres de service social diocésains reprochent aux religieuses de continuer à considérer leurs maisons d'hébergement comme leurs œuvres propres et d'accepter des enfants qu'on vient leur remettre directement. Ils voudraient que les orphelinats ne soient plus qu'une pièce d'un système de placement entièrement sous leur responsabilité : une organisation cohérente du service social diocésain, n'est-ce pas la meilleure garantie que l'Église puisse présenter à l'État pour tenter de garder la maîtrise du bienêtre social? Ils réussissent à en persuader le ministère: en mai 1960, de nouvelles directives retirent, au bénéfice des CSS, toutes leurs prérogatives aux directions des institutions ${ }^{59}$.

Quel rôle alors pour celles-ci? Cette question taraude Caritas-Canada, le Conseil canadien du bien-être social et bien des promoteurs de l'éducation spécialisée. De nombreux prêtres et religieux, surtout parmi les travailleurs sociaux mais pas seulement, plaident désormais ouvertement non seulement pour une spécialisation toujours plus poussée des institutions, mais pour une sélection si stricte de leurs pensionnaires qu'elle aboutirait à en exclure tous les jeunes non gravement handicapés physiquement ou mentalement ${ }^{60}$. L'abbé Rivard n'est pas d'accord. Avec d'autres éducateurs, il constate certains problèmes récurrents reliés au placement familial: relations parfois difficiles entre parents nourriciers et enfants, franche réticence de nombreuses commissions scolaires à accueillir les jeunes $^{61}$. Sur toutes les tribunes, il continue de soutenir que

57. R. Rivard, Les enfants privés de milieu familial normal sont, à ce titre, des enfants exceptionnels, mars 1963, dact., 14 p. (B. 8, ch. "Conseil du Québec de l'enfance exceptionnelle»).

58. Rapport Parent, 2: 72 .

59. s.a., Le rôle de l'institution pour enfants privées de famille-ce qu'en attendent l'Église et l'État, dact., s.d. [1960], 2 p. (B. 4, ch. "Enfants privés de famille - Institutions ou foyers nourriciers»); s.a., La protection de l'enfance dans le diocèse de Trois-Rivières, dact., 3 p. plus organigramme. (B. 3, ch. "Protection de l'enfance»). Les auteurs de ces deux textes sont probablement pour l'un, M" Bourgeois directeur du Centre de service social de Trois-Rivières, et pour l'autre Jules Perron, son adjoint.

60. Conférence sans titre de l'abbé Euchariste Paulhus dans Le spécifique de l'éducateur spécialisé ou Qu'est-ce que l'éducateur spécialisé?, 27 mai 1960, dact., 14 p. (B. 8, ch. "Éducation de l’enfance exceptionnelle. Formation des éducateurs»).

61. Ministère du Bien-être social, Service de l'enseignement, Rapport d'enquêtes sur quelques foyers nourriciers, 7 novembre 1960, 6 p., voir note 27. Ce problème traverse toutes les années 1950 et 1960 et on en trouve pas moins d'une vingtaine d'échos dans le fonds Rivard. Andrée Dufour, Histoire de l'éducation au Québec (Montréal, Boréal Express, 1997), 92. 
des institutions repensées et bien financées, complétées peut-être par des foyers de groupes, peuvent être meilleures que le placement en foyer nourricier pour certains types d'enfants ${ }^{62}$. Les sœurs, quant à elles, restent silencieuses mais se sentent bousculées. Leurs œuvres ne leur appartiennent déjà pratiquement plus, et les enfants qu'elles y accueillent sont de plus en plus différents de ceux qu'elles recevaient encore naguère.

\subsection{Le dynamisme du secteur de l'éducation}

La Révolution tranquille, tous l'ont dit, a d'abord été celle de l'éducation, y compris de l'éducation des enfants exceptionnels. Le contexte international s'y prête, du reste. Le Bureau international d'éducation, l'UNESCO et le président Kennedy, entre autres, contribuent alors à faire de la déficience intellectuelle et du retard pédagogique des questions importantes pour les gouvernements ${ }^{63}$. Des études en France, aux États-Unis et ailleurs mettent crûment en lumière la charge que représente à terme, pour les sociétés de l'État-providence, l'insouciance avec laquelle elles traitent leurs enfants désavantagés, dont on estime le nombre au quart ou à la moitié, selon les critères retenus, de la population scolaire du pays ${ }^{64}$. Cette nouvelle prise de conscience internationale se répercute au Québec.

En 1962, le Conseil de l'instruction publique se dote d'une sous-commission de l'enseignement aux enfants exceptionnels présidée par Clément Thibert, qui, on s'en souvient, a travaillé longtemps avec l'abbé Rivard dans les orphelinats de Trois-Rivières. Thibert entreprend aussitôt de développer l'enseignement et l'éducation aux exceptionnels en dehors des juridictions du ministère de la Famille et du Bien-être social, ainsi que d'organiser la profession d'éducateur spécialisé ${ }^{65}$.

62. Échange de correspondance entre Réal Rouleau, secrétaire général adjoint de la division Famille et enfance $d u$ Conseil canadien du bien-être social et R. Rivard, dir. technique de l'Institut psycho-social, 22 février et 3 mars 1961 plus 6 pages de documents annexés, et Conseil canadien du bien-être social, division Famille et enfance, Aperçu du projet concernant le soin des enfants en institution, dact., 3 p (B. 9, ch. "Étude sur les institutions pour enfants au Canada»); R. Rivard, Les services auxiliaires nécessaires au développement équilibré des enfants privés de famille normale, voir note 27.

63. Brochure de la XXIII Conférence internationale de l'Instruction publique, Bureau International d'éducation (Genève) et UNESCO (Paris) (Genève, 1960), 15 p. (B. 7, ch. "Enfance déficiente»); Allocution du Président des États-Unis, John F. Kennedy au Congrès, Remarks on Proposed Measures To Combat Mental Illness and Mental Retardation, 5 février 1963, 14 p. (B. 6, cartable vert).

64. L'enfance exceptionnelle. Mémoire présenté... par le Conseil des œuvres de Montréal, avril 1962, 17 et ss.

65. Les renseignements sur Clément Thibert ont été puisés dans plusieurs documents conservés dans le fonds Reynald-Rivard, la plupart étant des rapports ou des lettres rédigés par Thibert, qui spécifie à quel titre il les écrit. On les retrouve essentiellement dans les b. 7, 8, 9 et 15 . 
Premier front, le développement des classes spéciales ${ }^{66}$. La sous-commission prépare un Guide pour encourager les commissions scolaires à ouvrir de telles classes, qui aideront les familles à garder leurs enfants mésadaptés ou handicapés. En effet, le gouvernement ne souhaite plus que ceux-ci soient aussi systématiquement orientés vers les orphelinats ordinaires ou les institutions spécialisées ${ }^{67}$. En 1966, on pourra évaluer à 1500 le nombre de classes spéciales sous la responsabilité des commissions scolaires et à 20000 les enfants qui les fréquentent, un essor énorme en quelques années à peine ${ }^{68}$. C'est notamment le cas en Mauricie, où l'Institut psycho-social et Reynald Rivard mettent leur expertise au service des commissions scolaires dans une telle mesure de soutien aux familles naturelles ${ }^{69}$.

Deuxième front: l'organisation de la profession. Cela fait longtemps qu'on y pense. Tout au long des années 1950, à la demande de l'UNESCO, le Bureau international catholique de l'enfance (BICE) et l'Association internationale des éducateurs de jeunes inadaptés ont réfléchi aux moyens d'imposer cette nouvelle profession et multiplié les enquêtes internationales sur le statut, les tâches, les salaires, les conditions de travail, la sélection et la formation initiale et continue des personnes qui s'occupent des enfants exceptionnels; de plus, ils ont établi les articles d'un code-cadre de déontologie à leur intention ${ }^{70}$. Au Québec, Caritas-Canada se fait le relais et un agent actif d'une telle réflexion. Les discussions sont longues, y compris sur le nom et la définition de la profession ${ }^{71}$ !

66. La Commission Parent estime à 300000 le nombre des jeunes inadaptés au Québec. Rapport Parent, 2: 372-374.

67. Rapport Parent, 2: 377 et ss.; Guide à l'adresse des commissions scolaires et institutions qui désirent instituer des services d'éducation pour enfants exceptionnels: déficients mentaux éducables, Québec, document à étudier à la réunion du 19 juin 1964, dact., 9 p. (B. 8, ch. "Sous-commission de l'enseignement aux enfants exceptionnels»).

68. Albini Girouard, tsp., "Éditorial», L'enfant exceptionnel, Revue du CQEE, 3,1 (septembre 1966).

69. M.-P. Malouin, L'univers des enfants en difficulté..., op. cit., 229; «La Mauricie encore à l'avantgarde. Des professionnels forment une association en vue de l'éducation des enfants exceptionnels ", Le Nouvelliste, 16 avril 1962, 3 et 20.

70. La documentation contenue dans les b. 1, 3, 4, 7 et 8 permet de reconstituer le fin détail de cette réflexion. Pour un résumé : Henri R. Ouellet, L'enfance exceptionnelle au plan international (B. 9, ch. " $2^{\text {ème }}$ congrès 1964, Conseil du Québec de l'enfance exceptionnelle»).

71. Projet de rapport de la 9 e réunion de la sous-commission "Institutions" de la commission "Enfance» de Caritas-Canada tenue au secrétariat national à Montréal, 18 octobre 1960, dact., 5 p. (B. 17, ch. "Cours pour éducatrices des orphelinats, U. d’Ottawa 1959-1964»); Projet de rapport de la 10éunion de la sous-commission "Institutions" de la commission "Enfance» de Caritas-Canada, 23 novembre 1960, dact., 5 p. (B. 9, ch. "Étude sur les institutions pour enfants au Canada»). 
Comme le révèlent les nombreux documents qu'il a conservés, Reynald Rivard participe de près aux travaux de ces organismes. Il favorise la différenciation des responsabilités des diverses catégories de professionnels engagés auprès des enfants, en quoi il voit le gage de la spécificité des tâches des éducateurs spécialisés; il plaide pour l'élaboration de standards pour les institutions, qui indiqueraient le personnel requis et la formation exigée. Pour lui, le bien des enfants internés passe par la professionnalisation des éducateurs spécialisés, condition désormais pour une meilleure appréciation sociale des services que peuvent rendre les institutions ${ }^{72}$.

On ne se surprend pas, dès lors, de constater qu'il répond rapidement à l'invitation lancée par Clément Thibert en 1962 de former une Association régionale des professionnels travaillant auprès des inadaptés, ni qu'il s'engage aussi résolument dans le Conseil du Québec de l'enfance exceptionnelle dès sa création en $1963^{73}$. Le pôle de gravité de l'éducation spécialisée serait-il en train de se déplacer du ministère de la Famille et du Bien-être social vers le Conseil de l'Instruction publique puis le nouveau ministère de l'Éducation? C'est du moins ce que souhaite explicitement la sous-commission de l'enfance exceptionnelle ${ }^{74}$.

Parallèlement, Clément Thibert travaille sur un troisième front: la formation des éducateurs spécialisés en orphelinat, qui s'occupent des jeunes en dehors des heures de classe. En 1962, la sous-commission de l'enseignement aux enfants exceptionnels forme un sous-comité pour élaborer un programme d'enseignement. Reynald Rivard en fait partie avec d'autres, dont Marie-Andrée Bertrand, directrice des centres de sociopédagogie du ministère de la Famille et du Bien-être social et Gilles Gendreau, du Centre de formation des éducateurs spécialisés. On réussit à s'entendre sur un programme s'étendant sur 2 ans à temps partiel, s'adressant aux titulaires d'une $11^{\mathrm{e}}$ année et comprenant 840 heures d'enseignement théorique et pratique plus 1200 heures de stage clinique. Les

72. R. Rivard, Formation des éducatrices spécialisées..., voir note 46; et Projet de rapport de la $11^{\mathrm{e}}$ réunion de la sous-commission "Institutions" de la commission "Enfance» de Caritas-Canada, tenue au secrétariat national à Montréal, 8 mai 1961, dact., 6 p. (B. 8, ch. «Projet de cours pour éducateurs spécialisés. Caritas-Canada, 1960-1961»).

73. Jusqu'en 1967, Reynald Rivard présentera pratiquement chaque année des exposés aux congrès de cet organisme et il présidera ses comités et commissions chargés notamment de l'éducation des exceptionnels et de la formation des éducateurs. Voir notamment la B. 6, cartable vert.

74. Mémoire du département de l'Instruction publique (section catholique) à la Commission royale d'enquête sur l'enseignement, section du Service de l'enseignement aux enfants exceptionnels, rédigé par Clément Thibert, conseiller technique, 5 avril 1962, dact., 10 p. plus annexes. (B. 8, ch. "Éducation de l'enfance exceptionnelle. Formation des éducateurs»). 
matières couvertes montrent que Rivard a su s'imposer: formation psycho-pédagogique incluant une bonne préparation en dynamique de groupe et en pédagogie des loisirs; formation professionnelle axée sur le travail en équipe, l'organisation des récréations et les normes professionnelles des institutions pour enfants; méthodologie des techniques d'éducation faisant une large place aux arts, aux travaux manuels, aux sports et aux loisirs ${ }^{75}$. Le programme est approuvé en 1964 par la sous-commission de l'enfance exceptionnelle. Il est dès lors enseigné au Centre des études universitaires de Trois-Rivières, né 3 ans plus tôt.

Victoire de Rivard? Non. Le programme n'offre en fait, selon les dires mêmes de Clément Thibert, que «le minimum essentiel de formation professionnelle pour les éducateurs de groupe». On le trouve sans doute trop centré sur le développement des habiletés propres à aider les jeunes à exprimer leur personnalité, et pas assez sur l'acquisition des méthodes psychologiques susceptibles de favoriser leur rééducation. Par ailleurs, les techniques de loisirs prennent beaucoup de place, au détriment des aspects plus théoriques - psychologie, étude de la législation et de l'administration du bien-être social, techniques de probation et des enquêtes sociales - enseignés dans les cours de socio-pédagogie ${ }^{76}$.

Le nouveau cours d'éducateur de groupe ne sera donc donné «d'abord» qu'à Trois-Rivières et au Mont-Providence, et en fait, il ne le sera jamais ailleurs; il ne débouche pas sur un brevet ou une autre reconnaissance délivrée par le ministère de l'Éducation même s'il compte près de 350 heures de plus que les cours de socio-pédagogie et exige le même niveau scolaire préalable ${ }^{77}$. Le Centre de formation des éducateurs spécialisés, lié au Centre d'orientation et à l'Université de Montréal ainsi que les centres de sociopédagogie gardent leurs propres programmes et leurs propres conceptions théoriques et pratiques de l'intervention auprès des jeunes ${ }^{78}$. Il n'y aura pas

75. Procès-verbal d'une réunion du sous-comité "Éducateurs de cadre»à Montréal, 4 mars 1963, 2 p. (B. 8, ch. «Éducateurs de cadre»); Cahier Cours éducateurs de groupe 1965-1966, Service de réhabilitation, Hôpital Mont-Providence, 1965-1966, 9 p. (B. 21, Vrac, $1^{\text {ère}}$ ).

76. Lettre de L.-P. Audet, dir. des centres de socio-pédagogie aux membres de la Commission des Écoles Normales, 19 octobre 1959 (B. 6, ch. «Nouveau programme des Écoles normales - Cours à l'étude»).

77. Dossier Bureau de l'enfance exceptionnelle, Mémoire sur le cours éducateurs de groupe - École Normale des Ursulines de Roberval, 6 juin 1966 (B. 7, ch. «Nouveau programme des écoles Normales - Projets»); Consultation éducateurs de groupe. Compte-rendu de la deuxième réunion tenue à Québec, 15 février 1966 (B. 15, Articles-thèse).

78. Gilles Gendreau, avec la coll. de Diane Métayer et André Lebon, L'action éducative. Pour qui? Pour quoi?, op. cit., 44 et ss; Lettre de Nicole Bourget, responsable du programme au Mont-Providence et Reynald Rivard, dir. de la section d'Éducateurs de groupe de l'École d'aide sociale de Trois-Rivières, 22 janvier 1965 (B. 15, duo tang gris). 
de formation uniforme. À son tour, d'ailleurs, le Conseil du Québec de l'enfance exceptionnelle tente sans succès en 1966 et en 1967 de trouver un terrain d'entente pour mettre fin aux "querelles de clochers $»^{79}$.

Ce n'est d'ailleurs pas la seule source de division entre éducateurs spécialisés. L’enfance exceptionnelle étant de plus en plus écartelée entre divers ministères, Éducation, Bien-être social et Famille, et même Santé pour les enfants les plus handicapés, une coordination des services concernés s'impose absolument, et ce, au plus haut niveau ${ }^{80}$. Mais là où un abbé Riendeau, par exemple, un frère Paul-Émile Parent ou le Rapport Parent verraient bien en maître d'œuvre le futur ministère de l'Éducation, l'Association des éducateurs spécialisés ne souhaite pas du tout que le ministère du Bien-être social et de la Famille perde toute juridiction sur les exceptionnels; le spécifique de ces enfants (et sans doute aussi celui de leurs éducateurs!) ne pèserait pas lourd, ils le craignent, dans les préoccupations de l'autre ministère ${ }^{81}$.

\subsection{La marginalisation définitive des orphelinats et le destin des formations conçues à Trois-Rivières}

Pendant ce temps, les religieuses se rendent bien compte que leur formation ne correspond pas aux nouveaux standards, établis en fonction d'exigences que les hommes sont mieux placés pour remplir. Les inscriptions chutent au cours de "mères de groupe ": 75 en 1964, un pic, mais seulement 57, 3 ans plus tard ${ }^{82}$. Les prospectus n'indiquent-ils pas en toutes lettres que «Les Mères de groupe peuvent agir comme assistantes des éducateurs de groupe ou les remplacer où il n'y en aurait pas. La formation de la mère de groupe est moins poussée que celle de l'éducateur de

79. La formation des maîtres et éducateurs pour exceptionnels au Québec dans la diversité de ses formules. Rivard participe à ce panel. L'expression «querelles de clochers» est de Michel Rheault. $4^{\mathrm{e}}$ congrès du CQEE; et Lettre de Jeannine Guindon, présidente de la table ronde sur "Les paliers de formation des maîtres " à R. Rivard, 24 novembre 1967. $5^{\mathrm{e}}$ congrès du CQEE (B. 6, cartable vert).

80. Rivard fait partie de ceux qui demandent une telle coordination. Les enfants privés de milieu familial normal sont, à ce titre, des enfants exceptionnels, voir note 62 .

81. Causerie de l'abbé Robert Riendeau, directeur du Conseil des cuvres, lors d'un dîner organisé par le Conseil des auvres à l'occasion du congrès du Conseil du Québec de l'enfance exceptionnel à l'Hôtel Reine-Élizabeth, le $1^{\mathrm{er}}$ nov. 1963, dact., 16 p (B. 8, ch. "Conseil du Québec de l'enfance exceptionnelle»); Exposé de Paul-Émile Parent, f.c., dir. général de l'École Mont St-Antoine, L'enfance exceptionnelle dans la cité, les commissions scolaires et les institutions, dact., 7 p. (B. 9, ch. " $2^{\mathrm{e}}$ congrès 1964, Conseil du Québec de l'enfance exceptionnelle»); Évelyne Dumas-Gagnon, «Un groupe d'éducateurs spécialisés espère voir le ministère de la Famille jouer un rôle de premier plan dans l'éducation des enfants exceptionnels ", Le Devoir, 7 septembre 1965, 10.

82. ADT (Trois-Rivières) Archives personnelles de sr Bernadette Lavergne, o.p., Cours de formation de Mères de groupe, 4 cahiers, 1964 à 1967. 
groupe; elle ne requiert pas la $11^{\mathrm{e}}$ année de scolarité ${ }^{83}$ ». Les Dominicaines obtiennent au moins que l'abbé Rivard monte pour elles une formation spécifique en dynamique de groupe, d'une durée de 30 heures; celle-ci n'est reconnue que par une simple attestation de l'Institut psycho-social ${ }^{84}$. En 1967, les mères de groupe diplômées veulent davantage: une formation préparatoire à celle conduisant au diplôme d'éducateurs de groupe ${ }^{85}$. Quelques-unes, cinq ou six, font d'ailleurs partie de la première cohorte de ce programme; les autres s'inquiètent de ne pouvoir acquérir les compétences requises pour prendre soin des enfants beaucoup plus difficiles qu'elles reçoivent désormais.

Mais dès 1965, les dés sont jetés. Le ministère de la Famille et du Bienêtre social a fait son choix: maintenir les enfants dans leur foyer ou les ramener aussi rapidement que possible dans une famille restaurée. Pour cela, augmenter les subventions aux agences sociales afin qu'elles embauchent davantage de travailleurs sociaux. Les institutions, qui coûtent cher selon le ministère, deviennent une solution de dernier recours, on les invite à se spécialiser encore davantage et à ne plus accepter que les cas vraiment très lourds ${ }^{86}$. En 1967, sur 35000 protégés au Québec, il n’en reste que 5000 dans les orphelinats. Et des orphelinats, il n'en reste que 57, qu'on parle de transformer pour faire place plutôt à des pavillons d'unités familiales ${ }^{87}$. Ville-Joie du Rosaire est fermé et vendu; le ministère compte transformer l'édifice en centre de psychiatrie infantile ${ }^{88}$. En 1968,

83. Prospectus de la section Éducateurs de groupe, École d'aide sociale de Trois-Rivières, 1964, 8 p. (B. 21, Vrac, $1^{\text {ère }}$ ).

84. Procès-verbaux de réunions tenues entre l'abbé $R$. Rivard et des religieuses dominicaines, 5 et 26 octobre 1965, 26 novembre 1965, et Attestation de participation de l'Institut psycho-social, 7 juin 1966 (B. 15, documents libres au début de la boîte).

85. $1^{\text {er }}$ colloque des éducateurs de groupe. Réunion des Mères de groupe, samedi le $1^{\text {er }}$ avril 1967, dact., 2 p. (B. 9, ch. «Éducateurs de cadre»).

86. «La réorganisation du ministère de la Famille et du Bien-être social touchera : les personnes âgées, l'enfance exceptionnelle, les handicapés, le placement des enfants ", La Presse, 13 mars 1965, 8; Gabriel Blanchard, f.c., responsable du secteur institutionnel au ministère de la Famille et du Bien-être social, Orphelinats d'aujourd'hui et de demain, communication au $3^{\mathrm{e}}$ congrès du CQEE, décembre 1965, dact., 3 p. (B. 6, cartable vert). Pour une analyse de ce choix, M.-P. Malouin, op. cit., 409-410.

87. "Sur 35000 enfants hors foyer, il s'en trouve encore 5000 dans les orphelinats», Le Petit Journal, 4 juin 1967. L'article rapporte les propos de Jean-Paul Cloutier, ministre du Bien-être social et de la Famille. Le journaliste ajoute que «Les orphelinats ne seront bientôt plus qu'un mauvais souvenir».

88. «Ville-Joie du Rosaire sera converti en Centre de psychiatrie infantile», Le Nouvelliste, 24 novembre 1967; «Nouvel institut psychiatrique au coût de \$1 000000 », Le Nouvelliste, 7 juin 1969. Ville-Joie du Rosaire deviendra un centre d'accueil et de formation pour les déficients mentaux de 3 à 18 ans. 
les cours de mères de groupe se donnent pour la dernière fois à TroisRivières; ils auront formé 80 diplômées, dont 79 religieuses de 13 congrégations et une laïque.

En 1969, Reynald Rivard quitte la prêtrise. Il entre comme professeur à l'Université du Québec à Trois-Rivières, qui voit le jour cette année-là ; il y met sur pied un programme en génagogie, basé sur la dynamique de groupe, et un autre en enfance exceptionnelle où les loisirs sont une préoccupation de recherche et d'enseignement. La même année, une technique d'enseignement spécialisé fait partie du patrimoine fondateur des programmes du nouveau Cégep de Trois-Rivières. L'Institut psycho-social est intégré à l'hôpital Sainte-Marie en $1974^{89}$.

\section{CONCLUSION}

Au terme de ce parcours de la carrière de l'abbé Reynald Rivard consacrée directement à l'enfance, trois constats principaux se dégagent.

Sur les origines de l'éducation spécialisée, d'abord. Il appert que le réseau constitué par Boscoville, le Centre d'orientation et l'Institut de psychologie de l'Université de Montréal (auquel s'associe l'Université de Sherbrooke) ne fut pas le seul berceau de ce nouveau champ d'intervention et de formation entre les années 1940 et la fin des années 1960. En fait, la réflexion était générale et puisait à des courants théoriques et de pratiques contrastés, et même opposés. La plupart des universités y ont participé ainsi que des écoles normales et d'autres institutions de même niveau. La demande de formation émanait de nombreux types d'établissements pour enfants et pour jeunes, qu'ils soient déficients, délinquants, mésadaptés ou souffrent d'autres affections. Et les religieuses, autant que les religieux ou les jeunes hommes laïques, ont voulu se donner de véritables compétences professionnelles. Si nous avons surtout pu mettre en valeur la contribution de Rivard et du réseau trifluvien dont il est la charnière à l'essor de la discipline et de la profession, il faut souhaiter que les autres foyers d'origine soient bientôt étudiés à leur tour.

89. Association canadienne pour la santé mentale, filiale de Trois-Rivières, Rapport du comité scientifique sur les ressources et les besoins en santé mentale de la région administrative $n^{\circ} 4$, mai 1966 , 75 p. (B. 15, documents libres au début de la boîte); Brochure souvenir Cours Mères de groupe. 10 ans 1958-1968, offerte par le CEU à tous les participants au $2^{\mathrm{e}}$ colloque des Mères de groupe (B. 16, chemise jaune); Prospectus de l'UQTR, Modules et programmes 1970-1971 (B. 15, Articles-thèse). Les cours de mères de groupe sont donnés à Montréal en 1969, puis on perd leur trace. Correspondance, procès-verbaux et convention de cession de l'Institut psycho-social à l'Hôpital Sainte-Marie, plusieurs documents du 2 août 1973 au 27 novembre 1974 (B. 9, ch. «Institut psycho-social/Hôpital Sainte-Marie). 
Le deuxième constat touche évidemment la déqualification subie par les religieuses, comme sœurs et comme femmes. L'historiographie du mouvement de professionnalisation avait déjà mis le fait en lumière, notamment pour le service social ${ }^{90}$. Nous avons documenté ici des facettes essentielles de ce processus : l'impossibilité pour Rivard de faire valider par un diplôme reconnu les formations qu'il a conçues à l'intention des religieuses ainsi que le refus opposé par Caritas-Canada, le ministère du Bien-être social puis, plus tard, par celui de l'Éducation à sa demande de tenir compte des conditions de travail ou de formation préalable des sœurs dans le mode de dispensation des programmes d'éducation spécialisée.

Les relations entre l'Église et l'État, auxquelles l'historiographie est attentive lorsqu'elle s'intéresse aux origines de l'État-providence au Québec, se sont doublées de rapports de force internes à l'une et l'autre institution, c'est le troisième constat auquel conduit la biographie professionnelle de Reynald Rivard. L’opposition entre les établissements d'hébergement et les centres diocésains de services sociaux a recoupé en partie les rivalités professionnelles entre éducateurs spécialisés et travailleurs sociaux. Celle qui a grandi entre le ministère de la Famille et du Bien-être social et celui de l'Éducation, qui finit par l'emporter, rend compte partiellement du fait que le Québec a finalement choisi de ne plus compter sur les orphelinats ordinaires: les enfants qui pouvaient être désinstitutionnalisés ont été rendus à leurs familles naturelles ou placés en foyers nourriciers et les plus atteints, internés dans des établissements spécialisés. Des institutions bien conçues, bien soutenues par les pouvoirs publics, bien pourvues en ressources professionnelles et soucieuses de favoriser l'épanouissement des jeunes en travaillant avec eux à long terme auraientelles pu, pour certaines catégories d'enfants, constituer une meilleure solution? Rivard en était convaincu. Qu'en pensent aujourd'hui les éducateurs spécialisés?

Enfin, cette biographie professionnelle de Reynald Rivard permet d'entrevoir à quel point le Québec catholique des années 1945-1960 participe à la réflexion internationale sur la professionnalisation du vaste champ de la protection de la jeunesse. Cette dimension, que nous avons à peine effleurée ici, pourrait faire l'objet d’une enquête spécifique. 